\title{
HILBERT-KUNZ FUNCTIONS AND FROBENIUS FUNCTORS
}

\author{
SHOU-TE CHANG
}

\begin{abstract}
We study the asymptotic behavior as a function of $e$ of the lengths of the cohomology of certain complexes. These complexes are obtained by applying the $e$-th iterated Frobenius functor to a fixed finite free complex with only finite length cohomology and then tensoring with a fixed finitely generated module. The rings involved here all have positive prime characteristic.

For the zeroth homology, these functions also contain the class of HilbertKunz functions that a number of other authors have studied.

This asymptotic behavior is connected with certain intrinsic dimensions introduced in this paper: these are defined in terms of the Krull dimensions of the Matlis duals of the local cohomology of the module. There is a more detailed study of this behavior when the given complex is a Koszul complex.
\end{abstract}

\section{INTRODUCTION}

Unless otherwise specified, throughout this paper all rings are commutative with identity, Noetherian, and all modules are unitary. We shall use $p$ to denote a positive prime integer. We shall use $e$ for a variable element of $\mathbb{N}$, the set of nonnegative integers, and $q$ for a variable element of the set $\left\{p^{e}: e \in \mathbb{N}\right\}$.

When $R$ has characteristic $p$, we can consider the Frobenius map $F_{R}$, which is the endomorphism of $R$ sending $r \in R$ to $r^{p}$. The Frobenius functor $\mathrm{F}_{R}$ is the covariant functor from the category of $R$-modules to the category of $S$-modules obtained by applying $S \otimes_{R}$ in the special case where $S=R$ and $R \rightarrow S$ is the Frobenius endomorphism $F_{R}$. If we take a free complex $G_{\bullet}$, then $\mathrm{F}_{R}\left(G_{\bullet}\right)$ may be thought of as obtained by raising all the entries in the matrices of the boundary maps to their $p$-th powers. Given an $R$-module $M$ and a free resolution $G_{\bullet}$ of $M$, we can also think of $\mathrm{F}_{R}(M)$ as $\mathrm{H}_{0}\left(\mathrm{~F}_{R}\left(G_{\bullet}\right)\right)$. We shall simply write $\mathrm{F}$ for $\mathrm{F}_{R}$ when there is no confusion. We will use $\mathrm{F}^{e}$ to denote the $e$-th iterated Frobenius functor.

In this paper, we will switch freely between (homological) complexes $G_{\bullet}$ and (cohomological) complexes $G^{\bullet}$. We will use the term "homology" also for "cohomology" whenever it is convenient and does not need emphasis. By a finite complex we mean a complex which has have only finitely many nonzero terms, and except in rare specified cases, we assume that $G_{0}$ (or $\left.G^{0}\right) \neq 0$ and $G_{i}$ (or $\left.G^{i}\right)=0$ for $i<0$. The length of a finite complex is the supremum of $\left\{i: G_{i}\right.$ (or $\left.\left.G^{i}\right) \neq 0\right\}$. By a free complex we mean a complex of finitely generated free modules.

Received by the editors August 20, 1995.

1991 Mathematics Subject Classification. Primary 13A35; Secondary 13D03, 13D05, 13D25, 13D45, 18G15, 18G40.

Part of this work was done at the University of Michigan. The author would like to thank Professor Melvin Hochster for his many useful suggestions. The author is also partially supported by a grant from the National Science Council of R. O. C. 
Let $R$ be a Noetherian ring of characteristic $p, M$ a finitely generated $R$-module and $G^{\bullet}$ a finite free complex of finite length homology. It is stated and proved explicitly in Hochster and Huneke's paper [HH3] and proved implicitly in P. Robert's paper $[\mathrm{R} 2]$ that

$$
\ell\left(\mathrm{H}^{i}\left(\mathrm{~F}^{e}\left(G^{\bullet}\right) \otimes M\right)\right)=O\left(q^{i}\right)
$$

In this paper, we intend to find a best possible bound for $\ell\left(\mathrm{H}^{i}\left(\mathrm{~F}^{e}\left(G^{\bullet}\right) \otimes M\right)\right)$. It is worth noting that the two proofs for $(*)$ are substantially different. The first utilizes techniques developed with tight closure theory (see [HH1], etc.). In this paper, we study very closely the spectral sequence of a certain double complex which is a modified version of the one in [R2].

Let $R$ be a Noetherian ring of characteristic $p, I$ be an ideal and $M$ a finitely generated $R$-module such that $M / I M$ is of finite length. Then the Hilbert-Kunz function of $M$ with respect to $I$ is defined to be the function sending $e$ to $\ell\left(M / I^{[q]} M\right)$, where $I^{[q]}=\left(i^{q}: i \in I\right)$ (see $\left.[\mathrm{K} 1],[\mathrm{K} 2]\right)$. These functions turn out to be very hard to understand. There are already some beautiful results, but many more questions remain open (cf. [K1], [K2], [Mo1], [Mo2], [HM], [Cc], [Ct]).

If $I=\left(x_{1}, \ldots, x_{n}\right)$, then $M / I^{[q]} M=\mathrm{H}_{0}\left(\mathrm{~F}^{e}\left(\mathrm{~K}_{\bullet}\left(x_{1}^{q}, \ldots, x_{n}^{q} ; R\right)\right) \otimes M\right)$, where $\mathrm{K}$ • is the Koszul complex. Evidence shows that it's easier to study the behavior of Hilbert-Kunz functions from this point of view, i.e., as part of a sequence of homology functions (see, for example, $[\mathrm{Ct}]$ ). This is one of the motivations for the problem in this paper.

In order to simplify notation, the cohomological complex $G^{\bullet}$ will be used instead of the homological complex $G_{\bullet}$.

We may replace $R$ by $R / \operatorname{Ann} M$ without affecting any issue. Since $\mathrm{H}^{i}\left(G^{\bullet}\right)$ has finite length for all $i$, we have that $\bigcup_{i} \operatorname{Ass~} \mathrm{H}^{i}\left(G^{\bullet}\right)$ is a finite set of maximal ideals of $R$, say $\bigcup_{i} \operatorname{Ass~}^{i}\left(G^{\bullet}\right)=\left\{m_{1}, \ldots, m_{k}\right\}$. If we take a prime $P \notin\left\{m_{1}, \ldots, m_{k}\right\}$, then $\left(G^{\bullet}\right)_{P}$ is split exact free. This implies that the complex $\mathrm{F}^{e}\left(G^{\bullet}\right)_{P}$ is split exact free for all $e$. Therefore, the complex $\left(\mathrm{F}^{e}\left(G^{\bullet}\right) \otimes M\right)_{P}$ is split exact for any prime $P \notin\left\{m_{1}, \ldots, m_{k}\right\}$. Hence,

$$
\ell\left(\mathrm{H}^{i}\left(\mathrm{~F}^{e}\left(G^{\bullet}\right) \otimes M\right)\right)=\sum_{j} \ell\left(\mathrm{H}^{i}\left(\mathrm{~F}^{e}\left(G^{\bullet} \otimes_{R} R_{m_{j}}\right) \otimes_{R_{m_{j}}} M_{m_{j}}\right)\right) .
$$

From this point on, we will assume $(R, m)$ is local without loss of generality.

By Monsky's results ([Mo1]) we have $\ell\left(M / I^{[q]}\right)=c_{I}(M) q^{\operatorname{dim} M}+O\left(q^{\operatorname{dim} M-1}\right)$, where $c_{I}(M)>0$. Moreover, $c_{I}(M)$ is a positive integer when $M$ is one-dimensional. By Seibert's result ([Sei]), for each $i$ there exists $c_{i}^{\prime}$ such that

$$
\ell\left(\mathrm{H}^{i}\left(\mathrm{~F}^{e}\left(G^{\bullet}\right) \otimes M\right)\right)=c_{i}^{\prime} q^{\operatorname{dim} M}+O\left(q^{\operatorname{dim} M-1}\right) .
$$

In $\S 1$ we set the notation and together with $(*)$ we state and modify these known results (see (1.4)-(1.6)). However, in this paper we try to find a better bound for the length of $\mathrm{H}^{i}\left(\mathrm{~F}^{e}\left(G^{\bullet}\right) \otimes M\right)$ than these, and we want to achieve more. For example, we will show that for each $i$ there exists $c_{i}$ such that

$$
\ell\left(\mathrm{H}^{i}\left(\mathrm{~F}^{e}\left(G^{\bullet}\right) \otimes M\right)\right)=c_{i} q^{i}+O\left(q^{i-1}\right)
$$

(Corollary (2.11)). For the rest of $\S 1$ we study the double complex in Discussion (1.7) very closely, and a better bound is achieved. In fact, if we let $\delta_{M}^{i}=$ 
$\sup _{\nu \leq i} \operatorname{dim} \mathrm{H}_{m}^{\nu}\left(M^{\wedge}\right)$, we will see that

$$
\ell\left(\mathrm{H}^{i}\left(\mathrm{~F}^{e}\left(G^{\bullet}\right) \otimes M\right)\right)=c_{i} q^{\delta_{M}^{i}}+O\left(q^{\delta_{M}^{i}-1}\right)
$$

for some $c_{i}$ (Proposition (2.16)). It is a simple fact that $\delta_{M}^{i} \leq i$, and often the inequality is strict. We will see that equality holds if and only if $M$ contains a submodule of dimension $i$ if and only if $c_{i}$ in (**) is positive (Corollary (2.10)). It does seem reasonable that in this situation the length of $\mathrm{H}^{i}\left(\mathrm{~F}^{e}\left(G^{\bullet}\right) \otimes M\right)$ should be as large as possible (cf. $(*))$.

Indeed, the ultimate goal is not only to find the best bound for the functions studied, but to investigate whether they have behavior similar to that of HilbertKunz functions. To be precise, we want to know if there exists $\delta^{i}\left(M ; G^{\bullet}\right)$ such that

$$
\ell\left(\mathrm{H}^{i}\left(\mathrm{~F}^{e}\left(G^{\bullet}\right) \otimes M\right)\right)=c_{i} q^{\delta^{i}\left(M ; G^{\bullet}\right)}+O\left(q^{\delta^{i}\left(M ; G^{\bullet}\right)-1}\right)
$$

for some $c_{i}>0$. In $\S 2$ we find some sufficient conditions for this to be true (see, for example, Corollary (2.20)). In particular, we will solve the problem for the zeroand one-dimensional cases and the two-dimensional regular local case. However, we will show by an example (see Discussion (2.24)) that the situation for the general complexes is a little bit more complex than we hope for. For this reason we devote $\S 3$ to Koszul complexes (which is really the most important case). We will show that the bound achieved in $\S 1$ is actually sharp for Koszul complexes. Hence $\delta^{i}\left(M ; G^{\bullet}\right)$ in (\#) should be $\delta_{M}^{i}$ when $G^{\bullet}$ is a Koszul complex. However, it remains open whether (\#) holds true for Koszul complexes. In $\S 4$ we look at a non-trivial example not covered by our results in $\S 2$. We hope this example can shed some light for this very complicated problem.

(1.1) Discussion. Let $(R, m)$ and $\left(R^{\prime}, m^{\prime}\right)$ both be local. If $R \rightarrow R^{\prime}$ is flat local and $m R^{\prime}$ is an ideal primary to the maximal ideal of $R^{\prime}$, then for any finite length $R$ module $M, \ell_{R}(M)$ is a constant times $\ell_{R^{\prime}}\left(R^{\prime} \otimes M\right)$. The reason is that if we take a primary filtration of $M, 0=M_{0} \subsetneq M_{1} \subsetneq \cdots \subsetneq M_{l}=M$, where $M_{i} / M_{i-1} \simeq R / m$, then $0=R^{\prime} \otimes M_{0} \subsetneq R^{\prime} \otimes M_{1} \subsetneq \cdots \subsetneq R^{\prime} \otimes M_{l}=R^{\prime} \otimes M$ is a filtration of $R^{\prime} \otimes M$ with factors $R^{\prime} \otimes R / m \simeq R^{\prime} / m R^{\prime}$. Therefore,

$$
\ell_{R^{\prime}}\left(R^{\prime} \otimes M\right)=\ell_{R^{\prime}}\left(R^{\prime} / m R^{\prime}\right) \ell_{R}(M) .
$$

Moreover, since the diagram

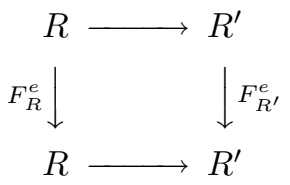

commutes for all $e$, it implies that $\mathrm{F}_{R^{\prime}}^{e}\left(R^{\prime} \otimes_{R} G^{\bullet}\right) \simeq R^{\prime} \otimes \mathrm{F}_{R}^{e}\left(G^{\bullet}\right)$. It follows that $\ell_{R^{\prime}}\left(\mathrm{H}^{i}\left(\mathrm{~F}_{R^{\prime}}^{e}\left(R^{\prime} \otimes_{R} G^{\bullet}\right) \otimes_{R^{\prime}}\left(R^{\prime} \otimes_{R} M\right)\right)\right)=\ell_{R^{\prime}}\left(R^{\prime} / m R^{\prime}\right) \ell_{R}\left(\mathrm{H}^{i}\left(\mathrm{~F}^{e}\left(G^{\bullet}\right) \otimes_{R} M\right)\right)$ for all $i$. Hence, in order to study the proposed problem, we can replace $G^{\bullet}$ by $R^{\prime} \otimes G^{\bullet}$ and $M$ by $R^{\prime} \otimes M$. In particular, we can let $R^{\prime}=\hat{R}$ and we may assume that $R$ is complete local.

If we assume $(R, m, k)$ is complete local, then $R$ contains a coefficient field $k$. Write $R$ as a module-finite extension over a power series $\operatorname{ring} A=k\left[\left[x_{1}, \ldots, x_{d}\right]\right]$. Let $k^{\prime}$ be the algebraic closure of $k$; then we can replace $R$ by $R \otimes_{A} B$ with $B=$ 
$k^{\prime}\left[\left[x_{1}, \ldots, x_{d}\right]\right]$ by the argument in the previous paragraph. Hence, we may assume that $k$ is perfect (or, even better, that $k$ is algebraically closed).

Denote by ${ }^{e} M$ the $R$-module which is $M$ as a set but the scalar multiplication "." is defined such that $x \cdot m=x^{q} m$ for all $x \in R$ and $m \in M$.

Let $I_{e}=\mathrm{Ann}^{e} M$. Then obviously we have $I_{0} \subseteq I_{1} \subseteq I_{2} \subseteq \cdots$. Since $R$ is Noetherian, there exists $e_{0}$ such that $I_{e_{0}}=I_{e_{0}+1}=I_{e_{0}+2}=\cdots$. Since we can assume $k$ is perfect, ${ }^{e_{0}} M$ is still a finitely generated $R$-module. The complex $\mathrm{F}^{e-e_{0}}\left(G^{\bullet}\right) \otimes{ }^{e_{0}} M$ is the same as ${ }^{e_{0}}\left(\mathrm{~F}^{e}\left(G^{\bullet}\right) \otimes M\right)$, so their homology modules have the same length. On the other hand, these homology modules have the same length as the homology of $\mathrm{F}^{e}\left(G^{\bullet}\right) \otimes M$, since $k$ is perfect. Hence, we may replace $M$ by ${ }^{e_{0}} M$ and assume that Ann $M=\operatorname{Ann}{ }^{1} M=\operatorname{Ann}^{2} M=\cdots$. Obviously, we may replace $R$ by $\bar{R}=R / \operatorname{Ann} M$ since $\mathrm{F}^{e}\left(G^{\bullet}\right) \otimes M \simeq \mathrm{F}_{\bar{R}}^{e}\left(G^{\bullet} \otimes \bar{R}\right) \otimes_{\bar{R}} M$. Thus, we may assume that ${ }^{e} M$ is faithful for all $e$. Let $N$ be the nilradical of $R$; then we can find $e^{\prime}$ such that $N^{q^{\prime}}=0$, where $q^{\prime}=p^{e^{\prime}}$. This says that $N \subseteq \operatorname{Ann}{ }^{e^{\prime}} M=0$, and so $R$ is reduced. Hence $R$ is reduced.

To sum up, we may assume that $M$ is faithful, while $R$ is complete local, reduced and its residue field is perfect (or, even better, algebraically closed).

(1.2) Discussion. Given a finite free complex

$$
G^{\bullet}=\left(0 \rightarrow G^{0} \stackrel{A_{1}}{\longrightarrow} G^{1} \rightarrow \cdots \stackrel{A_{n-1}}{\longrightarrow} G^{n-1} \stackrel{A_{n}}{\longrightarrow} G^{n} \rightarrow 0\right),
$$

we may assume that none of these maps is zero, for if not, we can just break it into shorter complexes and work on each segment of them. We may also assume $G^{\bullet}$ is minimal in the sense that $\operatorname{Im} A_{i} \subseteq m G^{i}$ for all $i$. Suppose $\operatorname{Im} A_{i} \not \subseteq m G^{i}$ for some $i$. Then we can do row and column operations so that $A_{i}$ looks like $\left(\begin{array}{ll}1 & \mathbf{0} \\ \mathbf{0} & \boldsymbol{\alpha}\end{array}\right)$, where $\boldsymbol{\alpha}$ is a $\left(\operatorname{rk} G^{i-1}-1\right) \times\left(\operatorname{rk} G^{i}-1\right)$-matrix. It follows that $A_{i-1}$ and $A_{i+1}$ will look like $\left(\begin{array}{ll}0 & * \\ \mathbf{0} & \boldsymbol{\beta}\end{array}\right)$ and $\left(\begin{array}{ll}0 & \mathbf{0} \\ * & \gamma\end{array}\right)$ respectively. This shows that $G^{\bullet}$ is the direct sum of a split exact sequence and the complex

$$
\cdots \stackrel{A_{i-2}}{\longrightarrow} G^{i-2} \stackrel{\left(\begin{array}{l}
* \\
\boldsymbol{\beta}
\end{array}\right)}{\longrightarrow} R^{\mathrm{rk} G^{i-1}-1} \stackrel{(\boldsymbol{\alpha})}{\longrightarrow} R^{\mathrm{rk} G^{i}-1} \stackrel{\left(\begin{array}{l}
* \\
\longrightarrow
\end{array}\right)}{\longrightarrow} G^{i+1} \stackrel{A_{i+2}}{\longrightarrow} \cdots,
$$

which is a complex different from $G^{\bullet}$ at only the $(i-1)$-th and the $i$-th terms and the three indicated boundary maps. We can repeat this process until eventually $G^{\bullet}$ becomes the direct sum of a minimal complex $G_{1}^{\bullet}$ and a split exact free complex $G_{2}^{\bullet}$. The complexes $\mathrm{F}^{e}\left(G_{2}^{\bullet}\right)$ and $\mathrm{F}^{e}\left(G_{2}^{\bullet}\right) \otimes M$ are again split exact, so $\mathrm{F}^{e}\left(G^{\bullet}\right) \otimes M$ and $\mathrm{F}^{e}\left(G_{1}^{\bullet}\right) \otimes M$ have the same homology. Hence, we may assume $G^{\bullet}$ is standard (see Definition 1.3).

(1.3) Definition. Let $(R, m)$ be a local ring. The finite free $R$-complex in $(*)$ is called standard if $A_{i}$ is non-zero and the entries of $A_{i}$ are in the maximal ideal $m$ for $i=1, \ldots, n$.

By standardizing a complex at the $i$-th spot we mean applying the process which transforms a non-standard complex into a standard complex as in Discussion (1.2). During the process, the complex might become a direct sum of shorter complexes. We shall retain only the segment containing the $i$-th spot and make an appropriate shift. 
(1.4) Proposition. Let $(R, m)$ a local ring of characteristic $p$ and $M$ be a finitely generated $R$-module of dimension $d$. If $\left(G_{\bullet}, d_{\bullet}\right)$ is a (possibly infinite) left free complex of finite length homology, then

$$
\ell\left(\mathrm{H}_{i}\left(\mathrm{~F}^{e}\left(G_{\bullet}\right) \otimes_{R} M\right)\right)=c_{i} q^{d}+O\left(q^{d-1}\right) \quad \text { for some real } c_{i} .
$$

Furthermore, if $G_{0} \neq 0$ and $d_{1}\left(G_{1}\right) \subset m G_{0}$, then $c_{0}>0$.

Proof. For the proof of the first part, see [Sei, Proposition 1]. To prove the second part, note that there is a copy of $M, M^{\prime}=M \oplus 0 \oplus \cdots \oplus 0$ inside $G_{0} \otimes M$. Denote the boundary maps of $\mathrm{F}^{e}\left(G_{\bullet}\right) \otimes M$ by $d_{\bullet}^{e}$. Then $\operatorname{Im}\left(d_{1}^{e}\right) \cap M^{\prime} \subset m^{q} M^{\prime}$. Hence

$$
\ell\left(\mathrm{H}_{i}\left(\mathrm{~F}^{e}\left(G_{\bullet}\right) \otimes_{R} M\right)\right) \geq \ell\left(\frac{M^{\prime}}{\operatorname{Im}\left(d_{1}^{e}\right) \cap M^{\prime}}\right) \geq \ell\left(\frac{M^{\prime}}{m^{q} M^{\prime}}\right) .
$$

The term on the far right is a polynomial in $q$ of degree $d$ for $e \gg 0$, so $c_{0}>0$.

(1.5) Corollary. Let $R$ be a local ring of characteristic $p, M$ a finitely generated $R$-module of dimension $d$ and $N$ a finite length $R$-module. Then

$$
\left.\ell\left(\mathrm{F}^{e}(N) \otimes_{R} M\right)\right)=c q^{d}+O\left(q^{d-1}\right) \quad \text { for some real } c>0 .
$$

Proof. Let $G$ • be a minimal free resolution of $N$. Then the result follows from Proposition 1.4.

(1.6) Theorem (P. Roberts). Let $R$ be a Noetherian ring of characteristic $p, M$ a finitely generated $R$-module of dimension $d$ and $G^{\bullet}$ a finite free complex of finite length homology. Then $\ell\left(\mathrm{H}^{i}\left(\mathrm{~F}^{e}\left(G^{\bullet}\right) \otimes_{R} M\right)\right)=O\left(q^{\min \{i, d\}}\right)$.

This result is implicitly given by $\mathrm{P}$. Roberts in his proof in [R2]. It is also explicitly given in $[\mathrm{HH} 3$, Theorem 6.2$]$ with a proof substantially different from that of P. Roberts. The new proof is a result of tight closure theory.

The double complex in Discussion 1.7 is a modification of the double complex P. Roberts used in [R2].

(1.7) Discussion. Let $R$ be a local ring and let $x_{1}, \ldots, x_{d}$ be a system of parameters for $R$. Consider the following double complex:

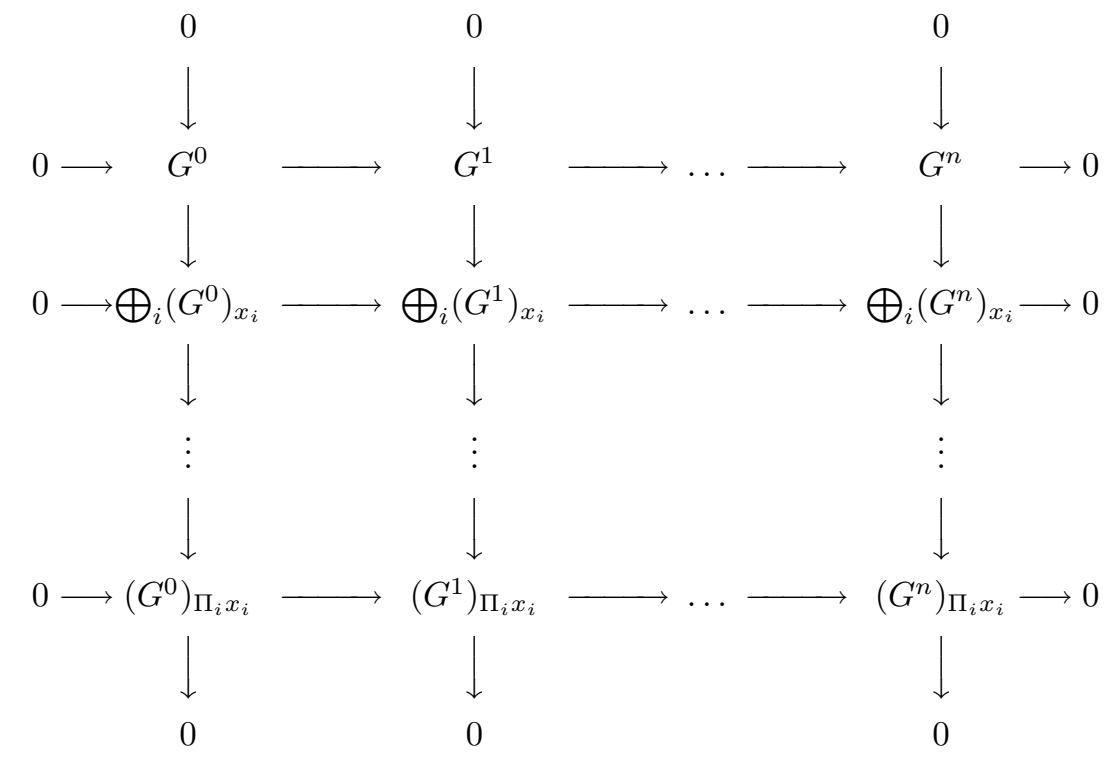


Let $M$ be a finitely generated $R$-module. By tensoring $M$ with this double complex, we get a new double complex $D=\left(D^{i j}, d^{i j}, e^{i j}\right)$, where $D^{i j}=\left(\underset{|S|=i}{\bigoplus_{1}}\left(G^{j}\right)_{\mathbf{x}(S)}\right) \otimes M$, the horizontal maps $d^{i j}$ are component maps and the vertical maps $e^{i j}$ are the differential maps in $\mathrm{K}^{\bullet}\left(\mathrm{x}^{\infty} ; G^{j} \otimes M\right)$. Take the homology of the rows. We can see that $\mathrm{H}_{\mathrm{II}}(D)$ is 0 except at the top row, in which $\mathrm{H}_{\mathrm{II}}(D)^{0, j}=\mathrm{H}^{j}\left(G^{\bullet} \otimes M\right)$. Hence,

$$
\mathrm{H}_{\mathrm{I}}\left(\mathrm{H}_{\mathrm{II}}(D)\right)^{i j}=\left\{\begin{array}{cc}
\mathrm{H}^{j}\left(G^{\bullet} \otimes M\right), & i=0, \\
0, & i \neq 0,
\end{array}\right.
$$

is already the $\mathrm{E}_{\infty}$-term. On the other hand, $\mathrm{H}_{\mathrm{II}}\left(\mathrm{H}_{\mathrm{I}}(D)\right)^{i j}=\mathrm{H}^{j}\left(\mathrm{H}_{m}^{i}(M) \otimes G^{\bullet}\right)$. Therefore,

$$
\mathrm{H}^{j}\left(G^{\bullet} \otimes \mathrm{H}_{m}^{i}(M)\right) \underset{j}{\Longrightarrow} \mathrm{H}^{i+j}\left(G^{\bullet} \otimes M\right) .
$$

(1.8) Lemma. Let $(R, m)$ be a local ring. A finite free $R$-complex

$$
G_{\bullet}=\left(\cdots \longrightarrow G_{i} \stackrel{A_{i}}{\longrightarrow} G_{i-1} \longrightarrow \cdots\right)
$$

is of finite length homology if and only if it satisfies the following two conditions:

(i) it satisfies the rank condition: $\operatorname{rk} G_{i}=\operatorname{rk} A_{i+1}+\operatorname{rk} A_{i}$ for all $i$, and

(ii) for all $i$, the rank ideal of $A_{i}$, the ideal generated by the ( $\left.\mathrm{rk} A_{i}\right)$-minors of $A_{i}$, is either the unit ideal or primary to $m$.

Proof. The complex $G_{\bullet}$ is of finite length homology if and only if for any $P \subsetneq m$,

$$
\mathrm{H}_{i}\left(\left(G_{\bullet}\right)_{P}\right)=\mathrm{H}_{i}\left(G_{\bullet}\right)_{P}=0 \quad \text { for all } i .
$$

Because $\left(G_{\bullet}\right)_{P}$ is a finite free $R_{P}$-complex, it is split exact. This happens exactly when $\left(G_{\bullet}\right)_{P}$ satisfies the rank condition and the rank ideals are the unit ideal of $R_{P}$. Hence the results.

(1.9) Remark. If $G^{\bullet}$ is a finite free complex of finite length homology, then so is $\operatorname{Hom}_{R}\left(G^{\bullet}, R\right)$.

(1.10) Definition. Let $(R, m, k)$ be a complete local ring, and $\mathrm{E}=\mathrm{E}_{R}(k)$ the injective hull of $k$. Define the $i$-th intrinsic module of $M$, denoted $M_{i}$, to be $\left(\mathrm{H}_{m}^{i}(M)\right)^{\vee}$, where ${ }^{\vee}=\operatorname{Hom}_{R}(-, \mathrm{E})$ is the Matlis dual.

(1.11) Lemma. If we let $G^{\prime} \bullet=\operatorname{Hom}_{R}\left(G^{\bullet}, R\right)$, then

$$
\mathrm{H}_{j}\left(G^{\prime} \bullet \otimes M_{i}\right) \underset{j}{\Longrightarrow}\left(\mathrm{H}^{i+j}\left(G^{\bullet} \otimes M\right)\right)^{\vee} .
$$

Proof. If we dualize the double complex $D$ in Discussion 1.7, then we get

$$
\mathrm{H}^{j}\left(G^{\bullet} \otimes \mathrm{H}_{m}^{i}(M)\right)^{\vee} \underset{j}{\Longrightarrow} \mathrm{H}^{i+j}\left(G^{\bullet} \otimes M\right)^{\vee} .
$$

Because the Matlis dual ${ }^{\vee}$ is an exact functor, we have

$$
\mathrm{H}^{j}\left(G^{\bullet} \otimes \mathrm{H}_{m}^{i}(M)\right)^{\vee} \simeq \mathrm{H}_{j}\left(\left(G^{\bullet} \otimes \mathrm{H}_{m}^{i}(M)\right)^{\vee}\right) .
$$

By the adjointness of $\otimes$ and Hom we have

$$
\left(G \bullet \otimes \mathrm{H}_{m}^{i}(M)\right)^{\vee}=\operatorname{Hom}_{R}\left(G^{\bullet}, \operatorname{Hom}_{R}\left(\mathrm{H}_{m}^{i}(M), E\right)\right)=G^{\prime} \bullet \otimes M_{i} .
$$

(1.12) Definition. Let $R$ be a complete local ring and $M$ a finitely generated $R$-module. The $i$-th intrinsic dimension of $M$, denoted $\delta_{M}^{i}$, is defined to be $\sup _{\nu \leq i} \operatorname{dim} M_{\nu}$, or in other words, the dimension of $M_{0} \oplus \cdots \oplus M_{i}$. 
(1.13) Proposition. Let $R$ be a local ring of characteristic $p, M$ a finitely generated $R$-module and $G^{\bullet}$ a finite free complex of finite length homology. Then

$$
\ell\left(\mathrm{H}^{i}\left(\mathrm{~F}^{e}\left(G^{\bullet}\right) \otimes M\right)\right)=O\left(q^{\delta_{M}^{i}}\right) .
$$

Proof. Let $G^{\prime} \bullet=\operatorname{Hom}_{R}\left(G^{\bullet}, R\right)$. Then obviously $\operatorname{Hom}_{R}\left(\mathrm{~F}^{e}\left(G^{\bullet}\right), R\right)=\mathrm{F}^{e}\left(G^{\prime} \bullet\right)$. The result follows from Remark (1.9), Lemma (1.11) and Proposition (1.4).

Next we define intrinsic modules in greater generality and explore some of their basic properties. Results throughout (1.14)-(1.22) are valid for all local rings. We give sketchy proofs; the reader can refer to [GH], [St] for more details.

(1.14) Lemma. Let $R$ be a local ring and $M$ a finitely generated $R$-module. If $S$ and $T$ are two Gorenstein rings such that $R=S / I=T / J$, then

$$
\operatorname{Ext}_{S}^{\operatorname{dim} S-i}(M, S) \simeq \operatorname{Ext}_{T}^{\operatorname{dim} T-i}(M, T)
$$

as $R$-modules.

Proof. Let $k$ be the residue field of $R, S$ and $T$. From local duality and adjointness of $\otimes$ and Hom one can easily check that both $\operatorname{Ext}_{S}^{\operatorname{dim} S-i}(M, S) \otimes_{R} \hat{R}$ and $\operatorname{Ext}_{S}^{\operatorname{dim} T-i}(M, T) \otimes_{R} \hat{R}$ are isomorphic to $\operatorname{Hom}_{R}\left(\mathrm{H}_{m}^{i}(M), \mathrm{E}_{R}(k)\right)$. Hence, $\operatorname{Ext}_{S}^{\operatorname{dim} S-i}(M, S) \simeq \operatorname{Ext}_{T}^{\operatorname{dim} T-i}(M, T)$ as $R$-modules.

(1.15) Definition. Let $R$ be a local ring and $M$ a finitely generated $R$-module. If $R=S / I$ with $S$ Gorenstein, define the $i$-th intrinsic module $M_{i}$ of $M$, denoted $M_{i}$, to be $\operatorname{Ext}_{R}^{\operatorname{dim} S-i}(M, S)$. In this case we can also define the $i$-th intrinsic dimension of $M$, denoted $\delta_{M}^{i}$, to be $\sup _{\nu \leq i} \operatorname{dim} M_{\nu}$, or in other words, the dimension of $\left(M_{0} \oplus \cdots \oplus M_{i}\right)$.

(1.16) Remark. A complete local ring $R$ is always the homomorphic image of a regular local ring, and thus its intrinsic modules always exist. In this case, the definition of the intrinsic modules coincides with Definition (1.12) by local duality.

(1.17) Remark. From the definition it is easy to see that the intrinsic dimensions form a non-decreasing sequence. However, we cannot say as much about the behavior of the dimensions of the intrinsic modules. In general, the dimensions of the intrinsic modules can be quite random, with only the limitation which we shall describe in Lemma (1.20).

(1.18) Lemma. Let $(R, m) \rightarrow(S, n)$ be a local homomorphism of local rings and let $S$ be module-finite over the image of $R$. If $M$ is a finitely generated $S$-module, then for each $i$, we can calculate $M_{i}$ either with respect to $R$ or with respect to $S$.

Proof. Let $K$ and $L$ be the residue fields of $R$ and $S$ respectively. Let $N$ be the $i$-th intrinsic module of $M$ as an $R$-module and $N^{\prime}$ be the $i$-th intrinsic module of $M$ as an $S$-module. Under this hypothesis, we can still use adjointness of $\otimes$ and Hom to get $N \otimes_{R} \hat{R} \simeq \operatorname{Hom}_{S}\left(\mathrm{H}_{n}^{i}(M), \mathrm{E}_{S}(L)\right) \simeq N^{\prime} \otimes_{S} \hat{S} \simeq N^{\prime} \otimes_{R} \hat{R}$. Hence $N \simeq N^{\prime}$.

(1.19) Lemma. Let $R$ be a local ring which is the homomorphic image of a Gorenstein ring, $M$ a finitely generated $R$-module and $P$ a prime ideal of $R$. Then $\left(M_{i}\right)_{P}=\left(M_{P}\right)_{i-\operatorname{dim} R / P}$, or, equivalently, $\left(M_{P}\right)_{i}=\left(M_{i+\operatorname{dim} R / P}\right)_{P}$. 
Proof. Let $R=S / J$, where $S$ is Gorenstein. If $Q$ is the contraction of $P$ in $S$ then $\operatorname{dim} S-\operatorname{dim} S_{Q}=\operatorname{dim} S-$ height $Q=\operatorname{dim} R / P$. Hence,

$$
\begin{aligned}
\left(M_{i}\right)_{P} & \simeq \operatorname{Ext}_{S}^{\operatorname{dim} S-i}(M, S)_{Q} \\
& \simeq \operatorname{Ext}_{S_{Q}}^{\operatorname{dim} S_{Q}+\operatorname{dim} R / P-i}\left(M_{P}, S_{Q}\right) \simeq\left(M_{P}\right)_{i-\operatorname{dim} R / P} .
\end{aligned}
$$

(1.20) Lemma. Let $R$ be a local ring which is the homomorphic image of a Gorenstein ring, and $M$ a finitely generated $R$-module. Then $\operatorname{dim} M_{i} \leq \delta_{M}^{i} \leq i$.

Proof. If $P \in \operatorname{Supp} M_{i}$ then by Lemma (1.19) we have $\left(M_{i}\right)_{P}=\left(M_{P}\right)_{i-\operatorname{dim} R / P} \neq 0$. This shows that $i \geq \operatorname{dim} R / P$. Hence,

$$
i \geq \max _{P \in \operatorname{Supp} M_{i}}\{\operatorname{dim} R / P\}=\operatorname{dim} M_{i} .
$$

(1.21) Lemma. Let $R$ be a local ring which is the homomorphic image of a Gorenstein ring, and $M$ a finitely generated $R$-module. Then $\operatorname{dim} M_{i}=\delta_{M}^{i}=i$ if and only if there exists $P \in \operatorname{Ass} M$ such that $\operatorname{dim} R / P=i$.

Proof. By Lemma (1.20), $\operatorname{dim} M_{i}=\delta_{M}^{i}=i$ if and only if there exists $P \in \operatorname{Supp} M_{i}$ with $\operatorname{dim} R / P=i$. By Lemma $(1.19),\left(M_{i}\right)_{P} \neq 0$ if and only if $\left(M_{P}\right)_{0} \neq 0$. This is equivalent to saying depth $M_{P}=0$, and hence equivalent to saying $P \in \operatorname{Ass} M$.

(1.22) Corollary. Let $R$ be a local ring which is the homomorphic image of a Gorenstein ring and $M$ a finitely generated $R$-module of dimension $d$. Then the lowest and the highest values of $i$ such that $M_{i} \neq 0$ are $\operatorname{depth} M$ and $d$ respectively. Moreover, $M_{d}$ is a non-zero module of dimension $d$.

Proof. The issue is not affected when we complete, so we may assume that $R$ is complete. Now this corollary is immediate from local duality, local cohomology and Lemma (1.21).

For definitions of spectral sequences the reader can refer to [Ser, Chapitre II.A, $6]$.

(2.1) Definition. Let $R$ be a complete local ring and $M$ be a finitely generated $R$-module. Let $\Delta(M ; i)=\left\{\nu \leq i: \operatorname{dim} M_{\nu}=\max \left\{0, \delta_{M}^{i}\right\}\right\}$.

When $R$ is just a local ring (not necessarily complete), define $\Delta(M ; i)$ to be $\Delta\left(M^{\wedge} ; i\right)$.

(2.2) Remark. By definition, the set $\Delta(M ; i)$ excludes all $\nu$ such that $M_{\nu}=0$. If $R$ is just any local ring, the intrinsic modules might not be defined. Definition (2.1) is an attempt to circumvent this inconvenience. Note that if $R$ is indeed a ring such that the intrinsic modules are defined, then since $\left(M^{\wedge}\right)_{\nu}=\left(M_{\nu}\right)^{\wedge}$ for all $\nu$, $\Delta(M ; i)$ is well-defined.

(2.3) Proposition. Let $R$ be a local ring of characteristic $p, M$ a finitely generated $R$-module and $G^{\bullet}$ a standard finite free complex of finite length homology.

1. If $\Delta(M ; i)=\varnothing$, then $\ell\left(\mathrm{H}^{i}\left(\mathrm{~F}^{e}\left(G^{\bullet}\right) \otimes M\right)\right)=0$ for all $e$. 
2. Suppose $|\Delta(M ; i)|=1$ and $\Delta(M ; i)=\left\{i_{0}\right\}$. Then there exists $c_{i}$ such that

$$
\ell\left(\mathrm{H}^{i}\left(\mathrm{~F}^{e}\left(G^{\bullet}\right) \otimes M\right)\right)=c_{i} q^{\delta_{M}^{i}}+O\left(q^{\delta_{M}^{i}-1}\right) .
$$

In fact, if we let $G^{\prime} \bullet=\operatorname{Hom}\left(G^{\bullet}, R\right)$ then

$$
c_{i}=\lim _{e \rightarrow \infty} \frac{\ell\left(\mathrm{H}^{i}\left(\mathrm{~F}^{e}\left(G^{\bullet}\right) \otimes M\right)\right)}{q^{\delta_{M}^{i}}}=\lim _{e \rightarrow \infty} \frac{\ell\left(\mathrm{H}_{i-i_{0}}\left(\mathrm{~F}^{e}\left(G^{\prime} \bullet\right) \otimes M_{i_{0}}\right)\right)}{q^{\delta_{M}^{i}}} .
$$

By Proposition (1.4), we know that $\lim _{e \rightarrow \infty} \frac{\ell\left(\mathrm{H}_{i-i_{0}}\left(\mathrm{~F}^{e}\left(G^{\prime} \bullet\right) \otimes M_{i_{0}}\right)\right)}{q^{\delta_{M}^{i}}}$ exists, positive or not.

Proof. We may assume that $R$ is complete. Part (1) is quite trivial. If $\Delta(M ; i)=$ $\varnothing$, we have $M_{0}=\cdots=M_{i}=0$. By Lemma (1.11), $\ell\left(\mathrm{H}^{i}\left(\mathrm{~F}^{e}\left(G^{\bullet}\right) \otimes M\right)\right)=$ $\ell\left(\left(\mathrm{H}^{i}\left(\mathrm{~F}^{e}\left(G^{\bullet}\right) \otimes M\right)\right)^{\vee}\right) \leq 0$. Now we can concentrate on the case where $|\Delta(M ; i)|=$ 1 and $\Delta(M ; i)=\left\{i_{0}\right\}$.

Fix $i$ and let $\delta_{M}^{i}=\alpha$. Consider the double complex $D$ in Discussion (1.7). Denote by ${ }^{e} \mathrm{E}_{r}$ the $\mathrm{E}_{r}$-term of the spectral sequence of the double complex obtained by applying $\mathrm{F}^{e}$ to the rows of $D$. By Lemma (1.11), the Matlis dual of the ${ }^{e} \mathrm{E}_{2}^{i j}$ term is $\mathrm{H}_{j}\left(\mathrm{~F}^{e}\left(G^{\prime} \bullet\right) \otimes M_{i}\right)$, so ${ }^{e} \mathrm{E}_{2}^{i j}$ and $\mathrm{H}_{j}\left(\mathrm{~F}^{e}\left(G^{\prime} \bullet\right) \otimes M_{i}\right)$ have the same length. Note that $\mathrm{d}_{r}^{e}:{ }^{e} \mathrm{E}_{r}^{i j} \rightarrow{ }^{e} \mathrm{E}_{r}^{i-r+1, j+r}$. By assumption, for all $s \leq i$ such that $s \neq i_{0}$ we have $\operatorname{dim} M_{s}<\alpha$. By Theorem (1.6), for all such $s$ and for all $t$,

$$
\ell\left({ }^{e} \mathrm{E}_{2}^{s t}\right)=\ell\left(\mathrm{H}_{t}\left(\mathrm{~F}^{e}\left(G^{\prime} \bullet\right) \otimes M_{s}\right)\right)=O\left(q^{\alpha-1}\right) .
$$

Hence, for $r \geq 2, t \geq 0$ and $s \leq i$ such that $s \neq i_{0}$, we have

$$
\ell\left({ }^{e} \mathrm{E}_{r}^{s t}\right)=O\left(q^{\alpha-1}\right) .
$$

This implies that for all $s \leq i$ such that $i \neq i_{0}$ we have $\ell\left({ }^{e} \mathrm{E}_{\infty}^{s, i-s}\right)=O\left(q^{\alpha-1}\right)$.

By Proposition (1.4), there exists $c$ such that

$$
\ell\left({ }^{e} \mathrm{E}_{2}^{i_{0}, i-i_{0}}\right)=\ell\left(\mathrm{H}_{i-i_{0}}\left(\mathrm{~F}^{e}\left(G^{\prime} \bullet\right) \otimes M_{i_{0}}\right)\right)=c q^{\alpha}+O\left(q^{\alpha-1}\right) .
$$

Now it suffices to show that $\ell\left({ }^{e} \mathrm{E}_{\infty}^{i_{0}, i-i_{0}}\right)=c q^{\alpha}+O\left(q^{\alpha-1}\right)$. We will show by induction

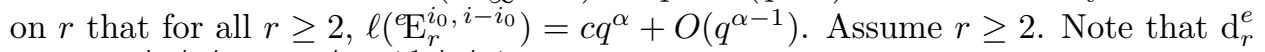
sends ${ }^{e} \mathrm{E}_{r}^{i_{0}, i-i_{0}}$ to ${ }^{e} \mathrm{E}_{r}^{i_{0}-r+1, i-i_{0}+r}$. Since $i_{0}-r+1<i_{0}$, by $(*)$, the length of its kernel is $c q^{\alpha}+O\left(q^{\alpha-1}\right)$. On the other hand, $\mathrm{d}_{r}^{e}$ sends ${ }^{e} \mathrm{E}_{r}^{i_{0}+r-1, i-i_{0}-r}$ to ${ }^{e} \mathrm{E}_{r}^{i_{0}, i-i_{0}}$. If $2 \leq r \leq i-i_{0}$ then

$$
i_{0}<i_{0}+r-1 \leq i_{0}+i-i_{0}-1=i-1,
$$

and we get $\ell\left({ }^{e} \mathrm{E}_{r}^{i_{0}+r-1, i-i_{0}-r}\right)=O\left(q^{\alpha-1}\right)$. If $r>i-i_{0}$ then $i-i_{0}-r<0$, and we get ${ }^{e} \mathrm{E}_{r}^{i_{0}+r-1, i-i_{0}-r}=0$. In either case we have $\ell\left(\mathrm{d}_{r}^{e}\left({ }^{e} \mathrm{E}_{r}^{i_{0}+r-1, i-i_{0}-r}\right)\right)=O\left(q^{\alpha-1}\right)$. Because ${ }^{e} \mathrm{E}_{r+1}^{i_{0}, i-i_{0}}$ is the homology of $\mathrm{d}_{r}^{e}$, we get $\ell\left({ }^{e} \mathrm{E}_{r+1}^{i_{0}, i-i_{0}}\right)=c q^{\alpha}+O\left(q^{\alpha-1}\right)$. This completes the induction step. Because $\bigoplus_{0}^{i} \mathrm{E}_{\infty}^{s, i-s}$ is an associated graded object of $\mathrm{H}^{i}\left(\mathrm{~F}^{e}\left(G^{\bullet}\right) \otimes M\right)$, we can conclude that

$$
\ell\left(\mathrm{H}^{i}\left(\mathrm{~F}^{e}\left(G^{\bullet}\right) \otimes M\right)\right)=\sum_{s=0}^{i} \ell\left({ }^{e} \mathrm{E}_{\infty}^{s, i-s}\right)=c q^{\alpha}+O\left(q^{\alpha-1}\right) .
$$


Note that

$$
\lim _{e \rightarrow \infty} \frac{\ell\left(\mathrm{H}^{i}\left(\mathrm{~F}^{e}\left(G^{\bullet}\right) \otimes M\right)\right)}{q^{\alpha}}=c=\lim _{e \rightarrow \infty} \frac{\ell\left(\mathrm{H}_{i-i_{0}}\left(\mathrm{~F}^{e}\left(G^{\prime} \bullet\right) \otimes M_{i_{0}}\right)\right)}{q^{\alpha}} .
$$

(2.4) Corollary. Let $R$ be a local ring of characteristic p, $M$ a finitely generated $R$-module and $G^{\bullet}$ a standard complex of finite length homology. If $\Delta(M ; i)=\{i\}$, then $\ell\left(\mathrm{H}^{i}\left(\mathrm{~F}^{e}\left(G^{\bullet}\right) \otimes M\right)\right)=c q^{\delta_{M}^{i}}+O\left(q^{\delta_{M}^{i}-1}\right)$ for some $c>0$. In fact,

$$
c=\lim _{e \rightarrow \infty} \frac{\ell\left(\mathrm{H}_{0}\left(\mathrm{~F}^{e}\left(G^{\prime} \bullet\right) \otimes M_{i}\right)\right)}{q^{\delta_{M}^{i}}},
$$

where $G^{\prime} \bullet=\operatorname{Hom}\left(G^{\bullet}, R\right)$.

Proof. The results follow from Proposition (2.3)(2) and Proposition (1.4).

(2.5) Corollary. Let $R$ be a local ring (of any characteristic), $M$ a finitely generated $R$-module and $G^{\bullet}$ a standard finite free complex of finite length homology. Then depth $M=\min \left\{\nu: \mathrm{H}^{\nu}\left(G^{\bullet} \otimes M\right) \neq 0\right\}$.

Proof. Let $h=\operatorname{depth} M$. Since $h$ is the smallest $\nu$ such that $M_{\nu} \neq 0$, it is easy to see that for all $s<h$ and all $t$, we get $\mathrm{E}_{2}^{s t}=0$, which implies $\mathrm{E}_{\infty}^{s t}=0$, and also that $\mathrm{E}_{\infty}^{h 0} \simeq \mathrm{E}_{2}^{h 0} \neq 0$.

(2.6) Discussion. In order to simplify notation, we use the extended real number system with the obvious arithmetic. This way when $\ell\left(\mathrm{H}^{i}\left(\mathrm{~F}^{e}\left(G^{\bullet}\right) \otimes M\right)\right)=0$ for $e \gg 0$, we can say that there exists a positive real (or integer) $c$ such that

$$
\ell\left(\mathrm{H}^{i}\left(\mathrm{~F}^{e}\left(G^{\bullet}\right) \otimes M\right)\right)=c q^{-\infty}+O\left(q^{-\infty}\right) \quad \text { for } e \gg 0
$$

since this is trivially true for any $c$ we choose. In particular, we can use this convention when $\delta_{M}^{i}=-\infty$. In this case, we can say that there exists a positive real (or integer) $c$ such that

$$
\ell\left(\mathrm{H}^{i}\left(\mathrm{~F}^{e}\left(G^{\bullet}\right) \otimes M\right)\right)=c q^{\delta_{M}^{i}}+O\left(q^{\delta_{M}^{i}-1}\right) .
$$

(2.7) Lemma. Let $R$ be a local ring of characteristic p, $M$ a zero-dimensional $R$-module and $G^{\bullet}$ any finite free complex of finite length homology. Then for any $i$, $\ell\left(\mathrm{H}^{i}\left(\mathrm{~F}^{e}\left(G^{\bullet}\right) \otimes_{R} M\right)\right)$ is eventually an integer valued constant function. Moreover, if $G^{\bullet}$ is standard of length $n$ then $\ell\left(\mathrm{H}^{i}\left(\mathrm{~F}^{e}\left(G^{\bullet}\right) \otimes_{R} M\right)\right)$ is eventually a positive integer-valued constant function for $0 \leq i \leq n$.

Proof. We may assume that $G^{\bullet}$ is standard. We can replace $R$ by $R / \operatorname{Ann} M$, and therefore we may assume that $R$ is Artinian. Because every element in the maximal ideal of $R$ is nilpotent, the differential maps in $\mathrm{F}^{e}\left(G^{\bullet}\right)$ all eventually become zero maps. Hence, $\ell\left(\mathrm{H}^{i}\left(\mathrm{~F}^{e}\left(G^{\bullet}\right) \otimes_{R} M\right)\right)=\left(\operatorname{rk} G^{i}\right) \ell(M)>0$ for $e \gg 0$.

(2.8) Remark. This lemma says that in the zero-dimensional case, for each $i$, there exist $d_{i} \in\{0,-\infty\}$ and a positive integer $c_{i}$ such that for $e \gg 0$,

$$
\ell\left(\mathrm{H}^{i}\left(\mathrm{~F}^{e}\left(G^{\bullet}\right) \otimes_{R} M\right)\right)=c_{i} q^{d_{i}}+O\left(q^{d_{i}-1}\right) .
$$

In particular, when $G^{\bullet}$ is standard of length $n$, for $0 \leq i \leq n, \delta_{M}^{i}=0$. In this case, for each such $i$, there exists a positive integer $c_{i}$ such that

$$
\ell\left(\mathrm{H}^{i}\left(\mathrm{~F}^{e}\left(G^{\bullet}\right) \otimes_{R} M\right)\right)=c_{i} q^{\delta_{M}^{i}}+O\left(q^{\delta_{M}^{i}-1}\right) .
$$

This completes the discussion of the zero-dimensional case. 
(2.9) Corollary. Let $R$ be a local ring of characteristic $p, M$ a finitely generated $R$-module and $G^{\bullet}$ a standard finite free complex of finite length homology and of length $n$. Then there exists a positive integer $c$ such that

$$
\ell\left(\mathrm{H}^{0}\left(\mathrm{~F}^{e}\left(G^{\bullet}\right) \otimes M\right)\right)=c_{i} q^{\delta_{M}^{0}}+O\left(q^{\delta_{M}^{0}-1}\right) .
$$

Proof. Consider the double complex $D$ in Discussion (1.7). Denote by ${ }^{e} \mathrm{E}_{r}$ the $\mathrm{E}_{r^{-}}$ term of the spectral sequence of the double complex obtained by applying $\mathrm{F}^{e}$ to the rows of $D$. Note that for $r \geq 2, \mathrm{~d}_{r}^{e}$ maps from ${ }^{e} \mathrm{E}_{r}^{00}$ to ${ }^{e} \mathrm{E}_{r}^{-r+1, r}=0$ and from ${ }^{e} \mathrm{E}_{r}^{r-1,-r}=0$ to ${ }^{e} \mathrm{E}_{r}^{00}$, so ${ }^{e} \mathrm{E}_{r+1}^{00} \simeq{ }^{e} \mathrm{E}_{r}^{00}$. Therefore, we have ${ }^{e} \mathrm{E}_{\infty}^{00} \simeq{ }^{e} \mathrm{E}_{2}^{00}$. Let $G^{\prime} \bullet=\operatorname{Hom}_{R}\left(G^{\bullet}, R\right)$. By Lemma (1.11),

$$
\ell\left(\mathrm{H}^{0}\left(\mathrm{~F}^{e}\left(G^{\bullet}\right) \otimes M\right)\right)=\ell\left({ }^{e} \mathrm{E}_{2}^{00}\right)=\ell\left(\left({ }^{e} \mathrm{E}_{2}^{00}\right)^{\vee}\right)=\ell\left(\mathrm{H}_{0}\left(\mathrm{~F}^{e}\left(G^{\prime} \bullet\right) \otimes M_{0}\right)\right) .
$$

Now the result follows from Proposition (1.4) and Discussion (2.6).

(2.10) Corollary. Let $R$ be a local ring of characteristic $p, M$ a finitely generated $R$-module and $G^{\bullet}$ a standard finite free complex of finite length homology. If there exists $P \in \operatorname{Ass} M$ such that $\operatorname{dim} R / P=i$, that is, if $M$ has a submodule of dimension $i$, then there exists $c_{i}>0$ such that

$$
\ell\left(\mathrm{H}^{i}\left(\mathrm{~F}^{e}\left(G^{\bullet}\right) \otimes M\right)\right)=c_{i} q^{i}+O\left(q^{i-1}\right) ;
$$

otherwise, $\ell\left(\mathrm{H}^{i}\left(\mathrm{~F}^{e}\left(G^{\bullet}\right) \otimes M\right)\right)=O\left(q^{i-1}\right)$. In particular, if $\operatorname{dim} M=d$, then

$$
\ell\left(\mathrm{H}^{d}\left(\mathrm{~F}^{e}\left(G^{\bullet}\right) \otimes M\right)\right)=c_{d} q^{d}+O\left(q^{d-1}\right) \quad \text { for some real } c_{d}>0 .
$$

Proof. By Lemma (1.21) the condition on $P$ implies that $\Delta(M ; i)=\{i\}$. Now the first part follows immediately from Corollary (2.4). The second part is a result of Lemma (1.21) and Proposition (1.13).

(2.11) Corollary. Let $R$ be a local ring of characteristic $p, M$ a finitely generated $R$-module and $G^{\bullet}$ a finite free complex of finite length homology. Then for all $i$,

$$
\ell\left(\mathrm{H}^{i}\left(\mathrm{~F}^{e}\left(G^{\bullet}\right) \otimes M\right)\right)=c_{i} q^{i}+O\left(q^{i-1}\right) .
$$

Proof. This is an immediate result of Corollary (2.10).

(2.12) Definition. Let $R$ be a local ring of characteristic $p, M$ a finitely generated $R$-module and $G^{\bullet}$ a finite free complex of finite length homology. For $0 \leq i \leq$ length of $G^{\bullet}$, we define

$$
\delta^{i}\left(M ; G^{\bullet}\right)=\left\{\begin{array}{l}
-\infty \quad \text { if } \ell\left(\mathrm{H}^{i}\left(\mathrm{~F}^{e}\left(G^{\bullet}\right) \otimes M\right)\right)=0 \text { for infinitely many } e \\
\sup \left\{\delta \in \mathbb{N}: \liminf _{e \rightarrow \infty} \ell\left(\mathrm{H}^{i}\left(\mathrm{~F}^{e}\left(G^{\bullet}\right) \otimes M\right)\right) / q^{\delta}>0\right\} \quad \text { otherwise }
\end{array}\right.
$$

(2.13) Discussion. Let $G^{\bullet}$ be standard. Let the dimension of $M$ be $d$ and let the length of $G^{\bullet}$ be $n$. From previous results we have when $\operatorname{dim} M=0$, when $i=0$, $d, n$ or when $i$ is such that $M$ has a submodule of dimension $i$, there exists $c_{i}>0$ such that

$$
\ell\left(\mathrm{H}^{i}\left(\mathrm{~F}^{e}\left(G^{\bullet}\right) \otimes M\right)\right)=c_{i} q^{\delta^{i}\left(M ; G^{\bullet}\right)}+O\left(q^{\delta^{i}\left(M ; G^{\bullet}\right)-1}\right),
$$

and in these cases we have $\delta^{i}\left(M ; G^{\bullet}\right)=\delta_{M}^{i}$ for $0 \leq i \leq n$. Hence, one natural question to ask is whether in general these functions have asymptotic behavior similar to that of Hilbert-Kunz functions. In other words, for each $i$, does there exist $c_{i}>0$ such that $(*)$ holds? 
We are also interested in the question of under what conditions is $\delta^{i}\left(M ; G^{\bullet}\right)$, whenever it is defined, independent of the choice of $G^{\bullet}$. One cannot expect complete independence of the choice of $G^{\bullet}$ without some restrictions. For example, let $R=$ $k[[x, y]]$ be the formal power series ring of two variables and let $\mathrm{K}^{\bullet}=\mathrm{K}^{\bullet}(x, y ; R)$. Let $G^{\bullet}=\mathrm{K}^{\bullet} \oplus \mathrm{K}^{\bullet-1}$ and $H^{\bullet}=\mathrm{K}^{\bullet} \oplus \mathrm{K}^{\bullet-2}$. It's easy to see that $\delta^{3}\left(M ; G^{\bullet}\right)=2$ while $\delta^{3}\left(M ; H^{\bullet}\right)=0$. Thus, we should consider at most only those standard complexes $G^{\bullet}$ that are not a nontrivial direct sum of finite free complexes. On the other hand, when $i=d$, we do have this independence. If we expect this property to be true, it's reasonable to suggest that $\delta^{i}\left(M ; G^{\bullet}\right)=d$ for $d \leq i \leq$ length of $G^{\bullet}$ under the mild restriction given above. We will see in Discussion (2.24) that unfortunately this is not true. It is hard to determine $\delta^{i}\left(M ; G^{\bullet}\right)$ in general. However, there is still no evidence that $\delta^{i}\left(M ; G^{\bullet}\right)$ should depend on the choice of $G^{\bullet}$ for $0 \leq i \leq d$.

Next we focus on the one-dimensional case. Proposition (2.16) tells what happens in the one-dimensional case.

(2.14) Definition. Let $R$ be a ring. Define $R^{\circ}$ to be the complement of the union of the minimal prime ideals of $R$.

(2.15) Lemma. Let $R$ be a Noetherian reduced ring of dimension $d$ and let $M$ be a finitely generated $R$-module. Then there exists a short exact sequence

$$
0 \rightarrow \bigoplus_{k} \frac{R}{P_{k}} \rightarrow M \rightarrow N \rightarrow 0,
$$

where the $P_{k}$ 's are the minimal primes of $R$ and $N$ has dimension less than $d$.

Proof. Let $P$ be a minimal prime of $R$. Since $R$ is reduced, $R_{P}=R_{P} / P R_{P}$ is a field. Thus, $M_{P}$ is a direct sum of $R_{P} / P R_{P}$. Let $n_{P} \in \mathbb{N}$ be such that $M_{P} \simeq\left(R_{P} / P R_{P}\right)^{n_{P}}$ and let

$$
L=\bigoplus_{P \text { minimal }}\left(\frac{R}{P}\right)^{n_{P}} .
$$

Then $\left(R^{\circ}\right)^{-1} L \simeq\left(R^{\circ}\right)^{-1} M$, and we can find $r \in R^{\circ}$ such that $r L \hookrightarrow M$. Since $R$ is reduced, $r$ is a nonzero-divisor of $R$. Hence, we can embed $L$ into $M$. Let $N$ be the cokernel. After localizing at a certain element in $R^{\circ}, L$ and $M$ become isomorphic, so $N$ is killed by an element in $R^{\circ}$. Hence, $\operatorname{dim} N<d$.

(2.16) Proposition. Let $R$ be a local ring of characteristic $p, M$ a finitely generated one-dimensional $R$-module and $G^{\bullet}$ a standard complex of finite length homology. Let the length of $G^{\bullet}$ be $n$. Then for $0 \leq i \leq n$ there exists a positive integer $c_{i}$ such that

$$
\ell\left(\mathrm{H}^{i}\left(\mathrm{~F}^{e}\left(G^{\bullet}\right) \otimes M\right)\right)=c_{i} q^{\delta_{M}^{i}}+O\left(q^{\delta_{M}^{i}-1}\right)
$$

(2.17) Remark. By Discussion (1.2) and Discussion (2.6), even when $G^{\bullet}$ is not standard, there exists a positive integer $c_{i}$ for each $0 \leq i \leq n$ such that

$$
\ell\left(\mathrm{H}^{i}\left(\mathrm{~F}^{e}\left(G^{\bullet}\right) \otimes M\right)\right)=c_{i} q^{\delta^{i}\left(M ; G^{\bullet}\right)}+O\left(q^{\delta^{i}\left(M ; G^{\bullet}\right)-1}\right) .
$$

We shall soon see that the $c_{i}$ 's involved here are all positive integers, and hence rationals. This is also true when $\operatorname{dim} M=0$ (Remark (2.8)). However, when $\operatorname{dim} M \geq 2$, the rationality conjecture remains open. 
Proof. The case $i=0$ follows from Corollary (2.9). We will assume $i \geq 1$. Notice that $\delta_{M}^{i}=1$ for $1 \leq i \leq n$.

By Discussion (1.1) we may assume that $R$ is complete and one-dimensional. Let

$$
G^{\bullet}=\left(0 \rightarrow G^{0} \stackrel{A_{1}}{\longrightarrow} G^{1} \stackrel{A_{2}}{\longrightarrow} \cdots \stackrel{A_{n}}{\longrightarrow} G^{n} \rightarrow 0\right) .
$$

Case 1. Suppose that $R$ is a P.I.D.

Let $r_{i}=\operatorname{rk} G^{i}$. We can do row and column operations so that

$$
A_{1}=\left(\begin{array}{ccc|c}
a_{1} & & \mathbf{0} & \mathbf{0} \\
\mathbf{0} & \ddots & & a_{r_{0}}
\end{array}\right)=\left(\begin{array}{ll}
B_{1} & \mathbf{0}
\end{array}\right) .
$$

This will force $A_{2}$ to look like $\left(\begin{array}{c}\mathbf{0} \\ A_{2}^{\prime}\end{array}\right)$, where $A_{2}^{\prime}$ is an $\left(r_{1}-r_{0}\right) \times r_{2}$ matrix. Now we can break up the original complex into the direct sum of the following two complexes:

$$
\begin{aligned}
0 \longrightarrow G^{0} \stackrel{B_{1}}{\longrightarrow} R^{r_{0}} \longrightarrow 0, \\
0 \longrightarrow R^{r_{1}-r_{0}} \stackrel{A_{2}^{\prime}}{\longrightarrow} G^{2} \stackrel{A_{3}}{\longrightarrow} \cdots .
\end{aligned}
$$

Since we assume that $A_{2} \neq 0$, we must have $r_{1}-r_{0} \neq 0$.

Repeat this process. The original complex becomes a direct sum of a number of Koszul complexes (of length 1) with proper shifting. In fact, there exist diagonal matrices $B_{1}, \ldots, B_{n}$ such that the complex $G^{\bullet}$ becomes the direct sum of the following complexes:

$$
\begin{aligned}
& 0 \longrightarrow R^{r_{0}} \stackrel{B_{1}}{\longrightarrow} R^{r_{0}} \longrightarrow 0, \\
& 0 \longrightarrow R^{r_{1}-r_{0}} \stackrel{B_{2}}{\longrightarrow} R^{r_{1}-r_{0}} \longrightarrow 0, \\
& 0 \longrightarrow R^{r_{2}-r_{1}+r_{0}} \stackrel{B_{3}}{\longrightarrow} R^{r_{2}-r_{1}+r_{0}} \longrightarrow 0 \text {, } \\
& \text { etc. }
\end{aligned}
$$

If we let $s_{i}=\sum_{j=0}^{i-1}(-1)^{i-j-1} r_{j}$ and let

$$
G_{j}^{\bullet}=\left(0 \rightarrow R^{s_{i}} \stackrel{B_{i}}{\longrightarrow} R^{s_{i}} \rightarrow 0\right),
$$

then, for $1 \leq i \leq n$,

$$
\ell\left(\mathrm{H}^{i}\left(\mathrm{~F}^{e}\left(G^{\bullet}\right) \otimes M\right)\right)=\ell\left(\mathrm{H}^{1}\left(\mathrm{~F}^{e}\left(G_{i}^{\bullet}\right) \otimes M\right)\right)+\ell\left(\mathrm{H}^{0}\left(\mathrm{~F}^{e}\left(G_{i+1}^{\bullet}\right) \otimes M\right)\right) .
$$

Note that $G_{i}^{\bullet}$ is a direct sum of standard Koszul complexes of length 1. Hence, $\ell\left(\mathrm{H}^{1}\left(\mathrm{~F}^{e}\left(G_{i}^{\bullet}\right) \otimes M\right)\right)$ is a sum of Hilbert-Kunz functions of dimension one. Since $\ell\left(\mathrm{H}^{0}\left(\mathrm{~F}^{e}\left(G_{i+1}^{\bullet}\right) \otimes M\right)\right)$ is bounded, we have $\ell\left(\mathrm{H}^{i}\left(\mathrm{~F}^{e}\left(G^{\bullet}\right) \otimes M\right)\right)=c_{i} q+O(1)$, where $c_{i}$ is a positive integer.

Case 2. Suppose that $R$ is a domain and $M=R$.

Let $R^{\prime}$ be the integral closure of $R$ in its field of fractions. Then $R^{\prime}$ is a local P. I. D. and we have a short exact sequence $0 \rightarrow R \rightarrow R^{\prime} \rightarrow N \rightarrow 0$, where $N$ is of 
dimension zero. This induces a long exact sequence

$$
\begin{aligned}
\cdots \rightarrow H^{i-1}\left(\mathrm{~F}^{e}\left(G^{\bullet}\right) \otimes N\right) \rightarrow & H^{i}\left(\mathrm{~F}^{e}\left(G^{\bullet}\right) \otimes R\right) \\
& \rightarrow H^{i}\left(\mathrm{~F}^{e}\left(G^{\bullet}\right) \otimes R^{\prime}\right) \rightarrow H^{i}\left(\mathrm{~F}^{e}\left(G^{\bullet}\right) \otimes N\right) \rightarrow \cdots .
\end{aligned}
$$

Hence for $1 \leq i \leq n$ we have $\ell\left(H^{i}\left(\mathrm{~F}^{e}\left(G^{\bullet}\right)\right)\right)=c_{i} q+O(1)$, where $c_{i}$ is a positive integer by Case 1 .

Case 3. The general case.

By Discussion (1.1) we may assume that $R$ is reduced. By Lemma (2.15) there exists a short exact sequence $0 \rightarrow \bigoplus_{k} R / P_{k} \rightarrow M \rightarrow N \rightarrow 0$ where $N$ is a finitelength module and the $P_{k}$ 's are such that $\operatorname{dim} R / P_{k}=1$. This short exact sequence induces a long exact sequence

$$
\begin{aligned}
\cdots \rightarrow H^{i-1}\left(\mathrm{~F}^{e}\left(G^{\bullet}\right) \otimes N\right) \rightarrow & H^{i}\left(\mathrm{~F}^{e}\left(G^{\bullet}\right) \otimes\left(\bigoplus_{k} \frac{R}{P_{k}}\right)\right) \\
& \rightarrow H^{i}\left(\mathrm{~F}^{e}\left(G^{\bullet}\right) \otimes M\right) \rightarrow H^{i}\left(\mathrm{~F}^{e}\left(G^{\bullet}\right) \otimes N\right) \rightarrow \cdots .
\end{aligned}
$$

The complex $G^{\bullet}$ is a standard $R$-complex of finite length homology, and this remains true for $\left(G^{\bullet} \otimes R / P_{k}\right)$ as an $\left(R / P_{k}\right)$-complex. Hence for $1 \leq i \leq n$ and for each $k$ we have $\ell\left(H^{i}\left(\mathrm{~F}^{e}\left(G^{\bullet}\right) \otimes R / P_{k}\right)\right)=c_{i}\left(R / P_{k}\right) q+O(1)$, where $c_{i}\left(R / P_{k}\right)$ is a positive integer. Thus

$$
\ell\left(H^{i}\left(\mathrm{~F}^{e}\left(G^{\bullet}\right) \otimes M\right)\right)=\left(\sum_{k} c_{i}\left(\frac{R}{P_{k}}\right)\right) q+O(1) .
$$

Next, we proceed to resolve the two-dimensional regular local case. But before doing so, we first show how to isolate the zeroth local cohomology module (as a submodule) from the module itself when computing our functions. We will also obtain results for the regular local case which shall explain how things go amok when we consider all finite free complexes of finite length homology.

(2.18) Lemma. Let $R$ be a local ring of characteristic $p, M$ a finitely generated $R$-module and $G^{\bullet}$ a standard complex of finite length homology. Let $D$ be the double complex in Discussion (1.7) and ${ }^{e} D$ the double complex obtained by applying $\mathrm{F}^{e}$ to the rows of D. Let $\mathrm{E}^{i j}$ and ${ }^{e} \mathrm{E}^{i j}$ be their spectral sequences respectively with $j$ as the filtration degree. Then for all $r \geq 1$ the map

$$
\mathrm{d}_{r}^{e}:{ }^{e} \mathrm{E}_{r}^{r-1, j} \rightarrow{ }^{e} \mathrm{E}_{r}^{0, j+r}
$$

is the zero map for $e \gg 0$.

Proof. Let

$$
\left\{\begin{array}{l}
\left({ }^{e} \mathrm{~K}^{n}\right)_{j}=\bigoplus_{\substack{s+t=n \\
t \geq j}}{ }^{e} D^{s t}, \\
{ }^{e} \mathrm{Z}_{r}^{i j}=\operatorname{Ker}\left(\left({ }^{e} \mathrm{~K}^{i+j}\right)_{j} \rightarrow\left({ }^{e} \mathrm{~K}^{i+j+1}\right)_{j+r}\right) .
\end{array}\right.
$$

An element $\bar{y}$ in ${ }^{e} \mathrm{E}_{r}^{r-1, j}$ is represented by an element $y$ in ${ }^{e} \mathrm{Z}_{r}^{r-1, j}$. By definition $y$ is an element in ${ }^{e} D^{r-1, j} \oplus \cdots \oplus{ }^{e} D^{0, j+r-1}$ which is mapped to an element $z$ in ${ }^{e} \mathrm{Z}_{r}^{0, j+r}={ }^{e} D^{0, j+r}$. The map d ${ }_{r}^{e}$ sends $\bar{y}$ to the image of $z$ in ${ }^{e} \mathrm{E}_{r}^{0, j+r}$. It suffices to show that $z=0$ for $e \gg 0$.

If $j+r>n$, then ${ }^{e} D^{0, j+r}=0$ and there is nothing to prove. We may assume that $j+r \leq n$, and in this case ${ }^{e} D^{0, j+r}=G^{j+r} \otimes M$. Because $z$ is the horizontal 
image (with respect to the diagram in Discussion (1.7)) of an element in ${ }^{e} D^{0, j+r-1}$, this shows that $z$ is in $G^{j+r} \otimes m^{[q]} M$. Because the vertical image (with respect to the diagram in Discussion (1.7)) of $z$ is 0 , we have that $z \in G^{j+r} \otimes \mathrm{H}_{m}^{0}(M)$. Hence $z \in G^{j+r} \otimes\left(m^{[q]} M \cap \mathrm{H}_{m}^{0}(M)\right)$. By the Artin-Rees Lemma, there exists $t$ such that $m^{[q]} M \cap \mathrm{H}_{m}^{0}(M) \subseteq m^{q} M \cap \mathrm{H}_{m}^{0}(M) \subseteq m^{q-t}\left(m^{t} M \cap \mathrm{H}_{m}^{0}(M)\right)=0$ for $e \gg 0$. Hence, $z$ has to be 0 and $\delta_{r}^{e}$ has to be the zero map for $e \gg 0$.

In the rest of the section, we will consider $\mathrm{H}_{m}^{0}(M)$ as a submodule of $M$ which consists of elements of $M$ that are killed by a power of $m$.

(2.19) Proposition. Let $(R, m)$ be a local ring of characteristic $p$ and $N$ the submodule $\mathrm{H}_{m}^{0}(M)$ of $M$. If $G^{\bullet}$ is a finite free complex of finite length homology, then

$$
\left.\ell\left(\mathrm{H}^{i}\left(\mathrm{~F}^{e}\left(G^{\bullet}\right) \otimes M\right)\right)\right)=\ell\left(\mathrm{H}^{i}\left(\mathrm{~F}^{e}\left(G^{\bullet}\right) \otimes N\right)\right)+\ell\left(\mathrm{H}^{i}\left(\mathrm{~F}^{e}\left(G^{\bullet}\right) \otimes M / N\right)\right)
$$

for each $i$.

Proof. We may assume $R$ is complete. Since $\mathrm{H}_{m}^{0}(M)=N=\mathrm{H}_{m}^{0}(N)$, so $M_{0}=$ $N^{\vee}=N_{0}$. If we let $G^{\prime} \bullet=\operatorname{Hom}\left(G^{\bullet}, R\right)$, then the $\mathrm{E}_{2}$-term of the dual of the double complex $D$ in Discussion (1.7) for $N$ has only one nonzero row: the top row. Hence $\ell\left(\mathrm{H}^{i}\left(\mathrm{~F}^{e}\left(G^{\bullet}\right) \otimes N\right)\right)=\ell\left(\mathrm{H}_{i}\left(\mathrm{~F}^{e}\left(G^{\prime} \bullet\right) \otimes N_{0}\right)\right)$. Let $x_{1}, \ldots, x_{d}$ be an s.o.p. for $R$. Write $\mathbf{x}(S)$ for $\prod_{i \in S} x_{i}$. For any nonempty subset $S$ of $\{1, \ldots, d\}$, we have $N_{\mathbf{x}(S)}=0$. Hence $M_{\mathbf{x}(S)} \simeq(M / N)_{\mathbf{x}(S)}$. This implies that the double complexes for $M$ and $M / N$ only differ at the top row. By Lemma (2.18) the ${ }^{e} \mathrm{E}_{\infty}$-terms of their spectral sequences for $M$ and $M / N$ only differ at the top row. Again, by Lemma (2.18) the ${ }^{e} \mathrm{E}_{\infty}$-term of $M$ has $\mathrm{H}_{j}\left(\mathrm{~F}^{e}\left(G^{\prime} \bullet\right) \otimes M_{0}\right)$ at the top row. On the other hand, we have depth $M / N>0$ and hence the ${ }^{e} \mathrm{E}_{\infty}$-term of $M / N$ has zeros at the top row. Hence

$$
\begin{aligned}
\left.\ell\left(\mathrm{H}^{i}\left(\mathrm{~F}^{e}\left(G^{\bullet}\right) \otimes M\right)\right)\right) & =\ell\left(\mathrm{H}_{i}\left(\mathrm{~F}^{e}\left(G^{\prime} \bullet\right) \otimes M_{0}\right)\right)+\ell\left(\mathrm{H}^{i}\left(\mathrm{~F}^{e}\left(G^{\bullet}\right) \otimes \frac{M}{N}\right)\right) \\
& =\ell\left(\mathrm{H}_{i}\left(\mathrm{~F}^{e}\left(G^{\prime} \bullet\right) \otimes N_{0}\right)\right)+\ell\left(\mathrm{H}^{i}\left(\mathrm{~F}^{e}\left(G^{\bullet}\right) \otimes \frac{M}{N}\right)\right) \\
& =\ell\left(\mathrm{H}^{i}\left(\mathrm{~F}^{e}\left(G^{\bullet}\right) \otimes N\right)\right)+\ell\left(\mathrm{H}^{i}\left(\mathrm{~F}^{e}\left(G^{\bullet}\right) \otimes \frac{M}{N}\right)\right) .
\end{aligned}
$$

(2.20) Corollary. Let $R$ be a local ring of characteristic $p, M$ a finitely generated $R$-module and $G^{\bullet}$ a standard complex of finite length homology. Let the length of $G^{\bullet}$ be $n$. Then the following are true for $0 \leq i \leq n$.

1. If $|\Delta(M ; i)-\{0\}| \leq 1$ then there exists $c_{i} \geq 0$ such that

$$
\ell\left(\mathrm{H}^{i}\left(\mathrm{~F}^{e}\left(G^{\bullet}\right) \otimes M\right)\right)=c_{i} q^{\delta_{M}^{i}}+O\left(q^{\delta_{M}^{i}-1}\right) .
$$

2. If $\Delta(M ; i) \subseteq\{0, i\}$ then there exists $c_{i}>0$ such that

$$
\ell\left(\mathrm{H}^{i}\left(\mathrm{~F}^{e}\left(G^{\bullet}\right) \otimes M\right)\right)=c_{i} q^{\delta_{M}^{i}}+O\left(q^{\delta_{M}^{i}-1}\right) .
$$

3. There exists $c_{1}>0$ such that

$$
\ell\left(\mathrm{H}^{1}\left(\mathrm{~F}^{e}\left(G^{\bullet}\right) \otimes M\right)\right)=c_{1} q^{\delta_{M}^{1}}+O\left(q^{\delta_{M}^{1}-1}\right) .
$$

Proof. Let $N=\mathrm{H}_{m}^{0}(M)$. The short exact sequence $0 \rightarrow N \rightarrow M \rightarrow M / N \rightarrow 0$ induces a long exact sequence

$$
\cdots \rightarrow N_{i+1} \rightarrow(M / N)_{i} \rightarrow M_{i} \rightarrow N_{i} \rightarrow \cdots .
$$

Hence $M_{i} \simeq(M / N)_{i}$ for $i>0$, while $(M / N)_{0}=0$. 
If $|\Delta(M ; i)-\{0\}| \leq 1$, then either $|\Delta(M ; i)| \leq 1$ or $\Delta(M ; i)=\left\{0, i_{0}\right\}$ is a two-element set. In the first case, part (1) follows from Proposition (2.3). In the second case, we have $|\Delta(M / N ; i)| \leq 1$. Hence part (1) follows from Proposition (2.19), Lemma (2.7) and Proposition (2.3)(2).

If $\Delta(M ; i)=\varnothing$ then the result follows from Discussion (2.6). If $\Delta(M ; i)=$ $\{i\}$ then the result follows from Corollary (2.4). If $\Delta(M ; i)=\{0\}$ or $\{0, i\}$, then either $\Delta(M / N ; i)=\varnothing$ or $\Delta(M / N ; i)=\{i\}$. Hence part (2) follows from Proposition (2.19), Discussion (2.6), Lemma (2.7) and Corollary (2.4).

Part (3) follows from part (2) since $\Delta(M ; 1) \subseteq\{0,1\}$.

The following two propositions deal with a special case when the homology of the complex in question is in some sense too large at a certain spot.

(2.21) Proposition. Let $(R, m)$ be a local ring of characteristic $p, M$ a finitely generated $R$-module of dimension d, and let $G \bullet$ be a (possibly infinite) free complex. Suppose

$$
G^{\bullet}=\left(\cdots \rightarrow G^{i-1} \stackrel{A_{i}}{\longrightarrow} G^{i} \stackrel{A_{i+1}}{\longrightarrow} G^{i+1} \rightarrow \cdots\right)
$$

where $A_{i}$ has entries in $m$ and $\operatorname{Ker}\left(A_{i+1}\right) \nsubseteq m G^{i}$. Then there exists $c>0$ such that $\ell\left(\mathrm{H}^{i}\left(\mathrm{~F}^{e}\left(G^{\bullet}\right) \otimes M\right)\right)=c q^{d}+O\left(q^{d-1}\right)$.

Proof. We already know that $c$ exists. We only need to prove that $c>0$. Under the hypotheses, we can find a direct summand $R$ of $G^{i}$ such that it maps into 0 . After a change of basis, we can assume that $A_{i+1}=\left(\begin{array}{c}\mathbf{0} \\ B\end{array}\right)$. Let $k=\operatorname{rk} G^{i}-1$; then we can rewrite $G^{\bullet}$ as

$$
\cdots \rightarrow G^{i-1} \stackrel{\left(\begin{array}{ll}
C & D
\end{array}\right)}{\longrightarrow} R \oplus R^{k} \stackrel{\left(\begin{array}{l}
\mathbf{0} \\
B
\end{array}\right)}{\longrightarrow} G^{i+1} \rightarrow \cdots .
$$

Because $\frac{R \oplus 0}{\operatorname{Im}(C D) \cap(R \oplus 0)}$ injects into $\mathrm{H}^{i}\left(G^{\bullet}\right)$, it has finite length. Since

$$
\operatorname{Im}(C D) \cap(R \oplus 0) \subset \operatorname{Im}(C) \oplus 0,
$$

we have

$$
\ell\left(\frac{R}{\operatorname{Im}(C)}\right) \leq \ell\left(\frac{R \oplus 0}{\operatorname{Im}(C D) \cap(R \oplus 0)}\right)<\infty .
$$

By Corollary (1.5) there exists $c^{\prime}>0$ such that $\ell\left(\mathrm{F}^{e}(R / \operatorname{Im} C) \otimes M\right)=c^{\prime} q^{d}+$ $O\left(q^{d-1}\right)$. Let ${ }^{e} C,{ }^{e} D$ be the matrices obtained by raising the entries in $C$ and $D$ to their $q$-th powers. Then

$$
\begin{aligned}
\ell\left(\mathrm{F}^{e}(R / \operatorname{Im} C) \otimes M\right) & =\ell\left(\frac{M}{\operatorname{Im}\left({ }^{e} C \otimes \operatorname{id}_{M}\right)}\right) \\
& \leq \ell\left(\frac{M \oplus 0}{\operatorname{Im}\left(\left({ }^{e} B C C^{e} D\right) \otimes \operatorname{id}_{M}\right) \cap(M \oplus 0)}\right) \\
& \leq \ell\left(\mathrm{H}^{i}\left(\mathrm{~F}^{e}\left(G^{\bullet}\right) \otimes M\right)\right) .
\end{aligned}
$$

Hence $c \geq c^{\prime}>0$.

(2.22) Proposition. Let $R$ be a d-dimensional regular local ring of characteristic $p, M$ a finitely generated $R$-module of the same dimension and $G^{\bullet}$ a finite free 
complex of finite homology. For each $i$ let $c_{i} \geq 0$ be the unique rational number such that $\ell\left(\mathrm{H}^{i}\left(\mathrm{~F}^{e}\left(G^{\bullet}\right) \otimes M\right)\right)=c_{i} q^{d}+O\left(q^{d-1}\right)$. Then $c_{i} \neq 0$ if and only if $\mathrm{H}^{i}\left(G^{\bullet}\right) \neq 0$.

Proof. Let

$$
G^{\bullet}=\left(\cdots \rightarrow G^{i-2} \stackrel{A_{i-1}}{\longrightarrow} G^{i-1} \stackrel{A_{i}}{\longrightarrow} G^{i} \rightarrow \cdots\right) .
$$

We can construct a new free complex

$$
\left(G^{\prime}\right)^{\bullet}=\left(\cdots \rightarrow\left(G^{\prime}\right)^{i-2} \stackrel{B_{i-1}}{\longrightarrow} G^{i-1} \stackrel{A_{i}}{\longrightarrow} G^{i} \rightarrow \cdots\right)
$$

such that the modules and maps in $\left(G^{\prime}\right)^{\bullet}$ are identical with those in $G^{\bullet}$ for degree no less than $i-1$ and $\mathrm{H}^{j}\left(\left(G^{\prime}\right)^{\bullet}\right)=0$ for $j<i$. One can do this by resolving $\operatorname{Ker} A_{i}$ over the regular local ring $R$. Now standardize $\left(G^{\prime}\right)^{\bullet}$ at the $i$ th spot and then shift the complex so that $\left(G^{\prime}\right)^{\bullet}$ is a right complex whose degree 0 piece is not zero. Let $l$ be such that $G^{\nu}=\left(G^{\prime}\right)^{\nu-l}$ for $\nu \geq i-1$. Then by construction $\left(G^{\prime}\right)^{\bullet}$ is still a complex of finite length homology and

$$
\mathrm{H}^{0}\left(\left(G^{\prime}\right)^{\bullet}\right)=\mathrm{H}^{1}\left(\left(G^{\prime}\right)^{\bullet}\right)=\cdots=\mathrm{H}^{i-1-l}\left(\left(G^{\prime}\right)^{\bullet}\right)=0 .
$$

Note that we have $\mathrm{H}^{i}\left(\mathrm{~F}^{e}\left(G^{\bullet}\right) \otimes M\right) \simeq \mathrm{H}^{i-l}\left(\mathrm{~F}^{e}\left(\left(G^{\prime}\right)^{\bullet} \otimes M\right)\right)$. Because $R$ is regular and all the rank ideals of $\left(G^{\prime}\right)^{\bullet}$ are $m$-primary ideals, by the Acyclicity Criterion (cf. $[\mathrm{BE}]$ or $[\mathrm{N}]), \mathrm{H}^{d}\left(\left(G^{\prime}\right)^{\bullet}\right)$ is the lowest nonzero homology of $\left(G^{\prime}\right)^{\bullet}$. By $(*)$, we have $i-l \leq d$, and $\mathrm{H}^{i}\left(G^{\bullet}\right) \neq 0$ if and only if $i-l=d$. Thus, $\mathrm{H}^{i}\left(G^{\bullet}\right) \neq 0$ if and only if $c_{i} \neq 0$ by Corollary (2.10).

The following corollary resolves the two-dimensional regular local case.

(2.23) Corollary. Let $R$ be a two-dimensional regular local ring of characteristic p, $M$ a finitely generated $R$-module and $G^{\bullet}$ a finite free complex of finite length homology and of length $n$. Then for $0 \leq i \leq n$ there exists $c_{i}>0$ such that

$$
\left.\ell\left(\mathrm{H}^{i}\left(\mathrm{~F}^{e}\left(G^{\bullet}\right) \otimes M\right)\right)\right)=c_{i} q^{\delta^{i}\left(M ; G^{\bullet}\right)}+O\left(q^{\delta^{i}\left(M ; G^{\bullet}\right)-1}\right) .
$$

Moreover, when $\operatorname{dim} M=2, \mathrm{H}^{i}\left(G^{\bullet}\right) \neq 0$ if and only if $\delta^{i}\left(M ; G^{\bullet}\right)=2$.

Proof. The zero-dimensional and one-dimensional cases are taken care of by Lemma (2.7) and Proposition (2.16). We will assume $\operatorname{dim} M=2$ and we will follow the same notation as in the proof of Proposition (2.22).

The case $\mathrm{H}^{i}\left(G^{\bullet}\right) \neq 0$ follows immediately from Proposition $(2.22)$. If $\mathrm{H}^{i}\left(G^{\bullet}\right)=0$, we can see from the proof of Proposition $(2.22)$ that $\left.\mathrm{H}^{i}\left(\mathrm{~F}^{e}\left(G^{\bullet}\right) \otimes M\right)\right)$ is isomorphic either to $\mathrm{H}^{0}\left(\mathrm{~F}^{e}\left(\left(G^{\prime}\right)^{\bullet} \otimes M\right)\right)$ or to $\mathrm{H}^{1}\left(\mathrm{~F}^{e}\left(\left(G^{\prime}\right)^{\bullet} \otimes M\right)\right)$. Now the result follows from Lemma (2.9) and Corollary (2.20)(3).

(2.24) Discussion. In Discussion (2.13) we asked if $\delta^{i}\left(M ; G^{\bullet}\right)$ is independent of $G^{\bullet}$ under the assumptions: (i) the length of $G^{\bullet}$ is no less than $i$ and (ii) the complex $G^{\bullet}$ is not a non-trivial direct sum of two finite free complexes of finite length homology. However, Proposition (2.22) shows that even with such very nice rings as regular local rings we cannot expect such a good property to be true.

For example, let $R=\mathbb{Z}_{p}[[x, y, z]]$ be a formal power series ring and let

$$
G^{\bullet}=\left(0 \rightarrow R \stackrel{A_{1}}{\longrightarrow} R^{3} \stackrel{A_{2}}{\longrightarrow} R^{3} \stackrel{A_{3}}{\longrightarrow} R^{3} \stackrel{A_{4}}{\longrightarrow} R^{3} \stackrel{A_{5}}{\longrightarrow} R \rightarrow 0\right)
$$


where

$$
\begin{aligned}
& A_{1}=\left(\begin{array}{lll}
z & -y & x
\end{array}\right), \quad A_{2}=A_{4}=\left(\begin{array}{ccc}
-y & x & 0 \\
-z & 0 & x \\
0 & -z & y
\end{array}\right), \\
& A_{3}=\left(\begin{array}{l}
x \\
y \\
z
\end{array}\right)\left(\begin{array}{lll}
z & -y & x
\end{array}\right) \text { and } A_{5}=\left(\begin{array}{l}
x \\
y \\
z
\end{array}\right) .
\end{aligned}
$$

We can also construct another complex

$$
H^{\bullet}=\left(0 \rightarrow H^{0} \stackrel{B_{1}}{\longrightarrow} H^{1} \stackrel{B_{2}}{\longrightarrow} H^{2} \stackrel{B_{3}}{\longrightarrow} H^{3} \stackrel{B_{4}}{\longrightarrow} R^{3} \stackrel{B_{5}}{\longrightarrow} R \rightarrow 0\right)
$$

where $B_{5}=A_{5}$ and

$$
B_{4}=\left(\begin{array}{ccc}
A_{5} & & \\
& A_{5} & \\
& & A_{5}
\end{array}\right)\left(\begin{array}{ccc}
-y & x & 0 \\
-z & 0 & x \\
0 & -z & y
\end{array}\right)
$$

while the rest of the complex is the minimal free resolution of Coker $B_{4}$. It is easy to see that neither $G^{\bullet}$ nor $H^{\bullet}$ can be written as a non-trivial direct sum of two shorter complexes. It is also easy to check that $\mathrm{H}^{4}\left(G^{\bullet}\right)=0$ while $\mathrm{H}^{4}\left(H^{\bullet}\right) \neq 0$. Obviously, we have $\mathrm{H}^{4}\left(\mathrm{~F}^{e}\left(G^{\bullet}\right)\right) \simeq \mathrm{H}^{2}\left(\mathrm{~F}^{e}\left(\mathrm{~K}^{\bullet}(x, y, z ; R)\right)\right)=0$ for all $e$. Thus, we have $\delta^{4}\left(R ; G^{\bullet}\right)=-\infty$ while $\delta^{4}\left(R ; H^{\bullet}\right)=3$.

In this example we can see that the independence of $G^{\bullet}$ fails for quite trivial reasons. However, it is possible that a finer analysis of the structure of the complex will give a better description of $\delta^{i}\left(M ; G^{\bullet}\right)$. Besides, there is no evidence that $\delta^{i}\left(M ; G^{\bullet}\right)$ should depend on $G^{\bullet}$ for $i \leq \operatorname{dim} M$. In fact, we know that $\delta^{i}\left(M ; G^{\bullet}\right)$ is independent of $G^{\bullet}$ for some special $i$ 's. However, as one can see here, working with all $G^{\bullet}$ does complicate matters. In the next section, we will concentrate on Koszul complexes.

For definitions and basic properties of Koszul complexes the reader can refer to [Mat1, 18D] or [Ser, Chapitre IV.A]. In this section we will show that $\delta^{i}\left(M ; \mathrm{K}^{\bullet}\right)=$ $\delta_{M}^{i}$ for all Koszul complexes $\mathrm{K}^{\bullet}$.

(3.1) Discussion. Let $x_{1}, \ldots, x_{n} \in R$. The Koszul complex $\mathrm{K}^{\bullet}\left(x_{1}, \ldots, x_{n} ; R\right)$ is defined to be $\mathrm{K}_{n-\bullet}\left(x_{1}, \ldots, x_{n} ; R\right)$, which is $\mathrm{K}_{\bullet}\left(x_{1}, \ldots, x_{n} ; R\right)$ numbered backward. But in essence

$$
\begin{aligned}
\mathrm{K}^{\bullet}\left(x_{1}, \ldots, x_{n} ; R\right) & \simeq \operatorname{Hom}_{R}\left(\mathrm{~K}_{\bullet}\left(x_{1}, \ldots, x_{n} ; R\right), R\right) \\
& =\mathrm{K}_{n-\bullet}\left((-1)^{n-1} x_{n},(-1)^{n-2} x_{n-1}, \ldots, x_{1} ; R\right) .
\end{aligned}
$$

Suppose $R$ is a ring containing a field $k$. The advantage of working with Koszul complexes is that we can assume that $R$ is regular by replacing $R$ by the polynomial ring $k\left[X_{1}, \ldots, X_{n}\right]$ (or the formal power series ring $k\left[\left[X_{1}, \ldots, X_{n}\right]\right]$ ), and $x_{1}, \ldots, x_{n}$ by $X_{1}, \ldots, X_{n}$.

(3.2) Lemma. Let $R$ be a local ring containing a field $k, M$ a finitely generated $R$-module and $x_{1}, \ldots, x_{n}$ be such that $\left(x_{1}, \ldots, x_{n}\right) R+\operatorname{Ann}_{R} M$ is primary to the 
maximal ideal. Let $l_{1}, \ldots, l_{n}$ be positive integers. Then

$$
\ell\left(\mathrm{H}_{i}\left(x_{1}^{l_{1}}, \ldots, x_{n}^{l_{n}} ; M\right)\right) \leq\left(\prod l_{k}\right) \ell\left(\mathrm{H}_{i}\left(x_{1}, \ldots, x_{n} ; M\right)\right) .
$$

Proof. Proving this lemma comes down to showing that

$$
\ell\left(\mathrm{H}_{i}\left(x_{1}^{a}, \ldots, x_{n} ; M\right)\right) \leq a \ell\left(\mathrm{H}_{i}\left(x_{1}, \ldots, x_{n} ; M\right)\right)
$$

for any positive integer $a$. We will show this by induction on $a$. This lemma is true for $a=1$. We now assume that $a>1$.

We may assume that $R=k\left[x_{1}, \ldots, x_{n}\right]$ is a polynomial ring. Because $x_{1}$ is a nonzero-divisor of $S=\frac{R}{\left(x_{2}, \ldots, x_{n}\right)}$, the sequence

$$
0 \rightarrow \frac{R}{\left(x_{1}^{a-1}, \ldots, x_{n}\right)} \rightarrow \frac{R}{\left(x_{1}^{a}, \ldots, x_{n}\right)} \rightarrow \frac{R}{\left(x_{1}, \ldots, x_{n}\right)} \rightarrow 0
$$

is exact and it induces a long exact sequence

$$
\begin{aligned}
\cdots \rightarrow \operatorname{Tor}_{i}^{R}\left(\frac{R}{\left(x_{1}^{a-1}, \ldots, x_{n}\right)}, M\right) \rightarrow \operatorname{Tor}_{i}^{R} & \left(\frac{R}{\left(x_{1}^{a}, \ldots, x_{n}\right)}, M\right) \\
& \rightarrow \operatorname{Tor}_{i}^{R}\left(\frac{R}{\left(x_{1}, \ldots, x_{n}\right)}, M\right) \rightarrow \cdots .
\end{aligned}
$$

In this situation Tor is the same as Koszul homology. The result follows from induction

(3.3) Remark. Let $R$ be a local ring containing a field, $M$ a finitely generated $R$ module and $x_{1}, \ldots, x_{n}$ be such that $\left(x_{1}, \ldots, x_{n}\right) R+\operatorname{Ann}_{R} M$ is primary to the maximal ideal. Let $l_{1}, \ldots, l_{n}$ be positive integers. Then

$$
\begin{aligned}
& \liminf _{e \rightarrow \infty} \frac{\ell\left(\mathrm{H}^{i}\left(\mathrm{~F}^{e}\left(\mathrm{~K}_{\bullet}\left(x_{1}^{l_{1}}, \ldots, x_{n}^{l_{n}} ; R\right)\right) \otimes M\right)\right)}{q^{s}}>0 \\
\Longleftrightarrow & \liminf _{e \rightarrow \infty} \frac{\ell\left(\mathrm{H}^{i}\left(\mathrm{~F}^{e}\left(\mathrm{~K}_{\bullet}\left(x_{1}, \ldots, x_{n} ; R\right)\right) \otimes M\right)\right)}{q^{s}}>0 .
\end{aligned}
$$

(3.4) Theorem. Let $(R, m)$ be a d-dimensional local ring of characteristic $p$ and $M$ be a finitely generated $R$-module. Suppose $x_{1}, \ldots, x_{n}$ generate an ideal primary to $m$. Then, for $0 \leq i \leq n$ such that $\delta_{M}^{i} \neq-\infty$,

$$
\liminf _{e \rightarrow \infty} \frac{\ell\left(\mathrm{H}^{i}\left(\mathrm{~F}^{e}\left(\mathrm{~K}^{\bullet}\left(x_{1}, \ldots, x_{n} ; R\right)\right) \otimes M\right)\right)}{q^{\delta_{M}^{i}}}>0 .
$$

Therefore, we have $\delta^{i}\left(M ; \mathrm{K}^{\bullet}\left(x_{1}, \ldots, x_{n} ; R\right)\right)=\delta_{M}^{i}$ even when $\delta_{M}^{i}=-\infty$.

Proof. The last statement follows from definition, Proposition (1.13) and the first part of this theorem.

We may assume that $\operatorname{dim} M=\operatorname{dim} R=d$ by replacing $R$ by $R / \operatorname{Ann} M$. We shall prove this theorem by induction on $\operatorname{dim} M$.

Lemma (2.7) takes care of the case $\operatorname{dim} M=0$. We may assume $\operatorname{dim} M=d>0$. Without loss of generality we may assume that $x_{1}, \ldots, x_{d}$ generate an ideal primary to $m$. In this case, there exists $s \in \mathbb{N}$ such that $x_{d+1}^{s}, \ldots, x_{n}^{s} \in\left(x_{1}, \ldots, x_{d}\right) R$. 
Moreover, we have $x_{d+1}^{q s}, \ldots, x_{n}^{q s} \in\left(x_{1}^{q}, \ldots, x_{d}^{q}\right) R$ for all $e$. One can easily check that

$$
\begin{aligned}
\mathrm{H}^{i}\left(\mathrm{~F}^{e}\right. & \left.\left(\mathrm{K}^{\bullet}\left(x_{1}, \ldots, x_{d}, x_{d+1}^{s}, \ldots, x_{n}^{s} ; R\right)\right) \otimes M\right) \\
= & \mathrm{H}^{i}\left(x_{1}^{q}, \ldots, x_{d}^{q}, x_{d+1}^{q s}, \ldots, x_{n}^{q s} ; M\right) \\
= & \bigoplus_{j=0}^{n-d}\left(\begin{array}{c}
n-d \\
j
\end{array}\right) \text { copies of } \mathrm{H}^{i-j}\left(x_{1}^{q}, \ldots, x_{d}^{q} ; M\right) \\
= & \bigoplus_{j=0}^{n-d}\left(\begin{array}{c}
n-d \\
j
\end{array}\right) \text { copies of } \mathrm{H}^{i-j}\left(\mathrm{~F}^{e}\left(\mathrm{~K}^{\bullet}\left(x_{1}, \ldots, x_{d} ; R\right)\right) \otimes M\right) .
\end{aligned}
$$

This implies that for $d \leq i \leq n$,

$$
\begin{gathered}
\liminf _{e \rightarrow \infty} \frac{\ell\left(\mathrm{H}^{i}\left(\mathrm{~F}^{e}\left(\mathrm{~K}^{\bullet}\left(x_{1}, \ldots, x_{d}, x_{d+1}^{s}, \ldots, x_{n}^{s} ; R\right)\right) \otimes M\right)\right)}{q^{d}} \\
\geq \liminf _{e \rightarrow \infty} \frac{\ell\left(\mathrm{H}^{d}\left(\mathrm{~F}^{e}\left(\mathrm{~K}^{\bullet}\left(x_{1}, \ldots, x_{d} ; R\right)\right) \otimes M\right)\right.}{q^{d}}>0 .
\end{gathered}
$$

Hence Remark (3.3) and Corollary (2.10) imply

$$
\liminf _{e \rightarrow \infty} \frac{\ell\left(\mathrm{H}^{i}\left(\mathrm{~F}^{e}\left(\mathrm{~K}^{\bullet}\left(x_{1}, \ldots, x_{n} ; R\right)\right) \otimes M\right)\right)}{q^{d}}>0
$$

for $d \leq i \leq n$. In fact, the argument above also shows that for $0 \leq i \leq d$ such that $\delta_{M}^{i} \neq-\infty$

$$
\begin{gathered}
\liminf _{e \rightarrow \infty} \frac{\ell\left(\mathrm{H}^{i}\left(\mathrm{~F}^{e}\left(\mathrm{~K}^{\bullet}\left(x_{1}, \ldots, x_{d}, x_{d+1}^{s}, \ldots, x_{n}^{s} ; R\right)\right) \otimes M\right)\right)}{q^{\delta_{M}^{i}}} \\
\quad \geq \liminf _{e \rightarrow \infty} \frac{\ell\left(\mathrm{H}^{i}\left(\mathrm{~F}^{e}\left(\mathrm{~K}^{\bullet}\left(x_{1}, \ldots, x_{d} ; R\right)\right) \otimes M\right)\right.}{q^{\delta_{M}^{i}}} .
\end{gathered}
$$

If we can prove the theorem for $\mathrm{K}^{\bullet}\left(x_{1}, \ldots, x_{d} ; R\right)$ for $0 \leq i<d$, then Remark (3.3) will imply the case for $\mathrm{K}^{\bullet}\left(x_{1}, \ldots, x_{n} ; R\right)$. Hence we may assume $n=d$. To sum up, we may assume that $R=k\left[\left[x_{1}, \ldots, x_{d}\right]\right]$ is regular local of dimension $d$, $\operatorname{dim} M=d$ and $\mathrm{K}^{\bullet}=\mathrm{K}^{\bullet}\left(x_{1}, \ldots, x_{d} ; R\right)$.

Since $R$ is reduced, by Lemma (2.15) there exists a short exact sequence

$$
0 \rightarrow \bigoplus R \rightarrow M \rightarrow N \rightarrow 0
$$

where $N$ is an $R$-module of dimension less than $d$. This short exact sequence induces a long exact sequence

$$
\cdots \rightarrow \bigoplus R_{i+1} \rightarrow N_{i} \rightarrow M_{i} \rightarrow \bigoplus R_{i} \rightarrow \cdots
$$

Since $R$ is Cohen-Macaulay of dimension $d$, this implies that for $i \leq d-2, N_{i} \simeq M_{i}$ and $\delta_{M}^{i}=\delta_{N}^{i}$. The induction hypothesis can be applied to $N$, so for $0 \leq i \leq d-2$ such that $\delta_{M}^{i} \neq-\infty$ we get

$$
\liminf _{e \rightarrow \infty} \frac{\ell\left(\mathrm{H}^{i}\left(\mathrm{~F}^{e}\left(\mathrm{~K}^{\bullet}\right) \otimes N\right)\right)}{q^{\delta_{M}^{i}}}>0 .
$$


The short exact sequence $(*)$ induces another long exact sequence

$$
\begin{aligned}
\cdots \rightarrow \mathrm{H}^{i}\left(\mathrm{~F}^{e}\left(\mathrm{~K}^{\bullet}\right) \otimes(\bigoplus R)\right) & \rightarrow \mathrm{H}^{i}\left(\mathrm{~F}^{e}\left(\mathrm{~K}^{\bullet}\right) \otimes M\right) \\
& \rightarrow \mathrm{H}^{i}\left(\mathrm{~F}^{e}\left(\mathrm{~K}^{\bullet}\right) \otimes N\right) \rightarrow \mathrm{H}^{i+1}\left(\mathrm{~F}^{e}\left(\mathrm{~K}^{\bullet}\right) \otimes(\bigoplus R)\right) \rightarrow \cdots .
\end{aligned}
$$

Since for $i \leq d-1$ we have $\mathrm{H}^{i}\left(\mathrm{~F}^{e}\left(\mathrm{~K}^{\bullet}\right) \otimes R\right)=0$, so $\mathrm{H}^{i}\left(\mathrm{~F}^{e}\left(\mathrm{~K}^{\bullet}\right) \otimes M\right) \simeq \mathrm{H}^{i}\left(\mathrm{~F}^{e}\left(\mathrm{~K}^{\bullet}\right) \otimes N\right)$ for $0 \leq i \leq d-2$. We now have shown that the theorem is true for $0 \leq i \leq d-2$. The only remaining case is when $i=d-1$. We may assume that $\delta_{M}^{d-1} \geq 0$.

Since $R$ is regular, we know $\mathrm{F}^{e}\left(G^{\bullet}\right)$ is a free resolution of $\frac{R}{\left(x_{1}, \ldots, x_{d}\right)^{[q]}}$ and

Take a minimal free $R$-resolution $F_{\bullet}$ of $M$, say

$$
\mathrm{H}^{i}\left(\mathrm{~F}^{e}\left(G^{\bullet}\right) \otimes M\right)=\operatorname{Tor}_{d-i}^{R}\left(\frac{R}{\left(x_{1}, \ldots, x_{d}\right)^{[q]}}, M\right) .
$$

$$
0 \longrightarrow F_{a} \stackrel{B_{a}}{\longrightarrow} \cdots \longrightarrow F_{2} \stackrel{B_{2}}{\longrightarrow} F_{1} \stackrel{B_{1}}{\longrightarrow} F_{0} \longrightarrow 0,
$$

where $a=\operatorname{pd} M$. If we let $\left(F^{\prime}\right)^{\bullet}=\operatorname{Hom}_{R}\left(F_{\bullet}, R\right)=$

$$
0 \longrightarrow F_{0} \stackrel{B_{1}^{\operatorname{tr}}}{\longrightarrow} F_{1} \stackrel{B_{2}^{\operatorname{tr}}}{\longrightarrow} \cdots \longrightarrow F_{a-1} \stackrel{B_{a}^{\operatorname{tr}}}{\longrightarrow} F_{a} \longrightarrow 0
$$

then by local duality we have $M_{i}=\operatorname{Ext}_{R}^{d-i}(M, R)=\mathrm{H}^{d-i}\left(\left(F^{\prime}\right)^{\bullet}\right)$.

Case 1. Suppose that $M$ has no submodule of dimension less than $\operatorname{dim} R$.

Since $M$ is torsion free, it is the first $R$-syzygy of a module $M^{\prime}$. Hence, we have $M_{i}^{\prime} \simeq M_{i+1}$ for $i \leq d-2$. Since $M_{0}=0$, we conclude that $\delta_{M^{\prime}}^{d-2}=\delta_{M}^{d-1}$. Furthermore, we have

$$
\begin{aligned}
\mathrm{H}^{d-1}\left(\mathrm{~F}^{e}\left(\mathrm{~K}^{\bullet}\right) \otimes M\right) & =\operatorname{Tor}_{1}^{R}\left(\frac{R}{\left(x_{1}, \ldots, x_{d}\right)^{[q]}}, M\right) \\
& =\operatorname{Tor}_{2}^{R}\left(\frac{R}{\left(x_{1}, \ldots, x_{d}\right)^{[q]}}, M^{\prime}\right) \\
& =\mathrm{H}^{d-2}\left(\mathrm{~F}^{e}\left(\mathrm{~K}^{\bullet}\right) \otimes M^{\prime}\right) .
\end{aligned}
$$

Since we already know the case for $i=d-2$, we have

$$
\liminf _{e \rightarrow \infty} \frac{\ell\left(\mathrm{H}^{d-1}\left(\mathrm{~F}^{e}\left(\mathrm{~K}^{\bullet}\right) \otimes M\right)\right)}{q^{\delta_{M}^{d-1}}}=\liminf _{e \rightarrow \infty} \frac{\ell\left(\mathrm{H}^{d-2}\left(\mathrm{~F}^{e}\left(\mathrm{~K}^{\bullet}\right) \otimes M^{\prime}\right)\right)}{q^{\delta_{M^{\prime}}^{d-2}}}>0 .
$$

Case 2. Let $N$ be any submodule of $M$ such that $M^{\prime}=M / N$ has no submodule of dimension less than $\operatorname{dim} M$. Suppose $\operatorname{dim} N<\delta_{M}^{d-1}$.

In this situation we have $\delta_{M}^{d-1}=\delta_{M^{\prime}}^{d-1}$. The short exact sequence

$$
0 \rightarrow N \rightarrow M \rightarrow M^{\prime} \rightarrow 0
$$

induces a long exact sequence

$$
\cdots \rightarrow N_{i+1} \rightarrow M_{i}^{\prime} \rightarrow M_{i} \rightarrow N_{i} \rightarrow \cdots
$$

Hence $M_{i} \simeq M_{i}^{\prime}$ for $i>\operatorname{dim} N$. The short exact sequence $(* *)$ induces another long exact sequence

$$
\cdots \rightarrow \mathrm{H}^{d-1}\left(\mathrm{~F}^{e}\left(\mathrm{~K}^{\bullet}\right) \otimes M\right) \rightarrow \mathrm{H}^{d-1}\left(\mathrm{~F}^{e}\left(\mathrm{~K}^{\bullet}\right) \otimes M^{\prime}\right) \rightarrow \mathrm{H}^{d}\left(\mathrm{~F}^{e}\left(\mathrm{~K}^{\bullet}\right) \otimes N\right) \rightarrow \cdots .
$$

It follows that

$$
\ell\left(\mathrm{H}^{d-1}\left(\mathrm{~F}^{e}\left(\mathrm{~K}^{\bullet}\right) \otimes M^{\prime}\right)\right) \leq \ell\left(\mathrm{H}^{d-1}\left(\mathrm{~F}^{e}\left(\mathrm{~K}^{\bullet}\right) \otimes M\right)\right)+\ell\left(\mathrm{H}^{d}\left(\mathrm{~F}^{e}\left(\mathrm{~K}^{\bullet}\right) \otimes N\right)\right)
$$


for all $e$. If we divide this inequality by $q^{\delta_{M}^{d-1}}$ we get

$$
\begin{aligned}
0 & <\liminf _{e \rightarrow \infty} \frac{\ell\left(\mathrm{H}^{d-1}\left(\mathrm{~F}^{e}\left(\mathrm{~K}^{\bullet}\right) \otimes M^{\prime}\right)\right)}{q^{\delta^{d-1}}} \\
& \leq \liminf _{e \rightarrow \infty}\left(\frac{\ell\left(\mathrm{H}^{d-1}\left(\mathrm{~F}^{e}\left(\mathrm{~K}^{\bullet}\right) \otimes M\right)\right)}{q^{\delta_{M}^{d-1}}}+\frac{\ell\left(\mathrm{H}^{d-1}\left(\mathrm{~F}^{e}\left(\mathrm{~K}^{\bullet}\right) \otimes N\right)\right)}{q^{\delta_{M}^{d-1}}}\right) \\
& =\liminf _{e \rightarrow \infty} \frac{\ell\left(\mathrm{H}^{d-1}\left(\mathrm{~F}^{e}\left(\mathrm{~K}^{\bullet}\right) \otimes M\right)\right)}{q^{\delta^{d-1}}} .
\end{aligned}
$$

Case 3. The only remaining case is when $M$ has a submodule of dimension $\delta_{M}^{d-1}$.

This case follows from the following lemma.

(3.5) Lemma. Let $(R, m)$ be a local ring of characteristic $p$ and $M$ be a finitely generated $R$-module. Let $x_{1}, \ldots, x_{n}$ generate an ideal primary to $m$. If $M$ contains a submodule $N$ of dimension $a$, then for $a \leq i \leq n$,

$$
\liminf _{e \rightarrow \infty} \frac{\ell\left(\mathrm{H}^{i}\left(\mathrm{~F}^{e}\left(\mathrm{~K}^{\bullet}\left(x_{1}, \ldots, x_{n} ; R\right)\right) \otimes M\right)\right)}{q^{a}}>0 .
$$

Proof. Let $\mathrm{K}^{\bullet}=\mathrm{K}^{\bullet}\left(x_{1}, \ldots, x_{n} ; R\right)$. Since $\operatorname{dim} N=a$, we may assume, by reordering if necessary, that there exists $s$ such that $x_{1}^{s}, \ldots, x_{n-a}^{s} \in\left(x_{n-a+1}, \ldots, x_{n}\right) R+$ Ann $N$. By Remark (3.3), we may assume that $x_{1}, \ldots, x_{n-a} \in \operatorname{Ann} N$.

Let $\mathrm{F}^{e}\left(\mathrm{~K}^{\bullet}\right)=\mathrm{K}_{e}^{\bullet}$ and let $\psi_{e}^{i}$ be the map from $\mathrm{K}_{e}^{i-1}$ to $\mathrm{K}_{e}^{i}$ in $\mathrm{F}^{e}\left(\mathrm{~K}^{\bullet}\right)$. Think of $\mathrm{K}^{\bullet}$ as $\mathrm{K}_{n-\bullet}$ and let $\left\{u_{j_{1}} \wedge \cdots \wedge u_{j_{n-i}}\right\}_{j_{1}<\cdots<j_{n-i}}$ generate $\mathrm{K}_{e}^{i}$. For any $m \in M$, $\psi_{e}^{i+1} \otimes \operatorname{id}_{M}$ sends $u_{1} \wedge \cdots \wedge u_{n-i} \otimes m$ to

$$
\sum_{j=1}^{i}(-1)^{j-1} x_{j}^{q}\left(u_{1} \wedge \cdots \wedge \widehat{u}_{j} \wedge \cdots \wedge u_{n-i}\right) \otimes m .
$$

For each $e$, there is a copy of $N$, say $N_{(e)}$, inside $R u_{1} \wedge \cdots \wedge u_{n-i} \otimes M \subset \mathrm{K}_{e}^{i} \otimes M$. When $a \leq i \leq n$ we have $0 \leq n-i \leq n-a$. Since $x_{1}^{q}, \ldots, x_{n-a}^{q} \in \operatorname{Ann} N$, this shows $N_{(e)}$ is inside the kernel of $\psi_{e}^{i+1} \otimes \operatorname{id}_{M}$ for all $e$. Thus,

$$
\frac{N_{(e)}}{\left(\psi_{e}^{i} \otimes \mathrm{id}_{M}\right)\left(\mathrm{K}_{e}^{i-1} \otimes M\right) \cap N_{(e)}} \hookrightarrow \mathrm{H}^{i}\left(\mathrm{~F}^{e}\left(\mathrm{~K}^{\bullet}\right) \otimes M\right) .
$$

There is an obvious surjection from $\frac{N_{(e)}}{\left(\psi_{e}^{i} \otimes \operatorname{id}_{M}\right)\left(\mathrm{K}_{e}^{i-1} \otimes M\right) \cap N_{(e)}}$ to $\frac{N}{m^{[q]} M \cap N}$.

By Artin-Rees Lemma, there exists a fixed $t$ such that for $q \gg 0$,

$$
m^{[q]} M \cap N \subseteq m^{q} M \cap N \subseteq m^{q-t}\left(m^{t} M \cap N\right) \subset m^{q-t} N .
$$

Therefore for $e \gg 0$,

$$
\begin{aligned}
\ell\left(\mathrm{H}^{i}\left(\mathrm{~F}^{e}\left(\mathrm{~K}^{\bullet}\right) \otimes M\right)\right) & \geq \ell\left(\frac{N}{m^{q-t} N}\right) \\
& =c(q-t)^{a}+\text { lower degree terms in } q, \quad \text { for some } c>0, \\
& =c q^{a}+\text { lower degree terms in } q .
\end{aligned}
$$

Hence,

$$
\liminf _{e \rightarrow \infty} \frac{\ell\left(\mathrm{H}^{i}\left(\mathrm{~F}^{e}\left(\mathrm{~K}^{\bullet}\right) \otimes M\right)\right)}{q^{a}}>c>0
$$


Let $(R, m)$ be a local ring of characteristic $p$ and $M$ be a finitely generated $R$-module. Let $x_{1}, \ldots, x_{n}$ generate an ideal primary to $m$. We would like to know whether there exists $c_{i}$ such that $\ell\left(\mathrm{H}^{i}\left(\mathrm{~F}^{e}\left(\mathrm{~K}^{\bullet}\right) \otimes M\right)=c_{i} q^{\delta_{M}^{i}}+O\left(q^{\delta_{M}^{i}-1}\right)\right.$ for $0 \leq i \leq n$. If this is true then by Theorem (3.4) we would have $c_{i}>0$. At this point this problem remains open. However, we do know when $|\Delta(M ; d-1)-\{0\}| \leq 1$ (see Corollary $(2.20)(1)$ ) this is true. One of the difficulties of this problem is the lack of examples. The examples either are too trivial or too complicated. In this section we calculate a non-trivial example in which the module is a module $M$ with $|\Delta(M ; d-1)-\{0\}|=2$.

(4.1) Example. Let $R=\mathbb{Z}_{p}\left[\left[x_{1}, \ldots, x_{d}\right]\right]$ be a formal power series ring. Let $\mathrm{K}_{\bullet}=\mathrm{K}_{\bullet}\left(x_{1}, \ldots, x_{d} ; R\right)$ and $\mathrm{K}^{\bullet}=\mathrm{K}^{\bullet}\left(x_{1}, \ldots, x_{d} ; R\right)$. Suppose $a_{1}, \ldots, a_{h}$ form a regular sequence in $R$ and $f_{1}, \ldots, f_{k} \in R-\{0\}$. Let $A$ be the $(h \times k)$-matrix $\left(a_{l} f_{m}\right)$ and let $M=\operatorname{Coker} A$.

When $d>h>1$ and $\operatorname{dim} \frac{R}{\sum_{m} f_{m} R}=d-h$, we will see that

$$
\Delta(M ; d-1)-\{0\}=\{d-h, d-1\}
$$

is a two-element set, and this case is not covered by Corollary $(2.20)(1)$. Nevertheless, we will show that in this case $\delta_{M}^{d-1}=d-h$ and

$$
\lim _{e \rightarrow \infty} \frac{\ell\left(\mathrm{H}^{d-1}\left(\mathrm{~F}^{e}\left(\mathrm{~K}^{\bullet}\right) \otimes M\right)\right)}{q^{d-h}}
$$

exists and is equal to

$$
\lim _{e \rightarrow \infty} \frac{\ell\left(\mathrm{H}_{0}\left(\mathrm{~F}^{e}\left(\mathrm{~K}_{\bullet}\right) \otimes M_{d-1}\right)\right)}{q^{d-h}}+\lim _{e \rightarrow \infty} \frac{\ell\left(\mathrm{H}_{h-1}\left(\mathrm{~F}^{e}\left(\mathrm{~K}_{\bullet}\right) \otimes M_{d-h}\right)\right)}{q^{d-h}} .
$$

In fact, assuming only the hypotheses in the first paragraph in this example, we claim that for each $0 \leq i \leq n$, there exists $c_{i}>0$ such that

$$
\ell\left(\mathrm{H}^{i}\left(\mathrm{~F}^{e}\left(\mathrm{~K}^{\bullet}\right) \otimes M\right)\right)=c_{i} q^{\delta_{M}^{i}}+O\left(q^{\delta_{M}^{i}-1}\right),
$$

and if $\delta_{M}^{i} \geq 0$ then

$$
c_{i}=\sum_{j=0}^{i} \lim _{e \rightarrow \infty} \frac{\ell\left(\mathrm{H}_{i-j}\left(\mathrm{~F}^{e}\left(\mathrm{~K}_{\bullet}\right) \otimes M_{j}\right)\right)}{q^{\delta_{M}^{i}}} .
$$

Since for $0 \leq j \leq i$ we have $\operatorname{dim} M_{i-j} \leq \delta_{M}^{i}$, the limit

$$
\lim _{e \rightarrow \infty} \frac{\ell\left(\mathrm{H}_{j}\left(\mathrm{~F}^{e}\left(\mathrm{~K}_{\bullet}\right) \otimes M_{i-j}\right)\right)}{q^{\delta_{M}^{i}}}
$$

exists by Proposition (1.4).

Proof of the claim. Let $e_{1}, \ldots, e_{k}$ be the standard free basis for $R^{k}$ and let $N$ be the quotient module of $R^{k}$ killing the submodule generated by the element $\left(f_{1}, \ldots, f_{k}\right)$ of $R^{k}$. The map from $M$ to $N$ sending the class of $e_{m}$ in $M$ to the class of $e_{m}$ in $N$ is well-defined. The kernel of this map is the cyclic submodule of $M$ generated by the class of $\left(f_{1}, \ldots, f_{k}\right)$. Let $L$ be this kernel. It is easy to see from the construction of $M$ that $L \simeq \frac{R}{\sum_{l} a_{l} R}$, so we have a short exact sequence

$$
0 \rightarrow L \rightarrow M \rightarrow N \rightarrow 0
$$


Because $a_{1}, \ldots, a_{h}$ is an $R$-sequence, $\mathrm{K}_{\bullet}\left(a_{1}, \ldots, a_{h} ; R\right)$ is a minimal free resolution of $L$. Hence

$$
L_{i} \simeq\left\{\begin{array}{cl}
0 & \text { if } i \neq d-h, \\
\frac{R}{\sum_{l} a_{l} R} & \text { if } i=d-h,
\end{array}\right.
$$

and $\frac{R}{\sum_{l} a_{l} R}$ has dimension $d-h$. Note that

$$
0 \rightarrow R \stackrel{\left(f_{1}, \ldots, f_{k}\right)}{\longrightarrow} R^{k} \rightarrow 0
$$

is a minimal free resolution of $N$. When $k=1$,

$$
N_{i} \simeq \begin{cases}0 & \text { if } i \neq d-1, \\ \frac{R}{f_{1} R} & \text { if } i=d-1,\end{cases}
$$

and $\operatorname{dim} N_{d-1}=d-1$. The short exact sequence $(*)$ induces a long exact sequence,

$$
\cdots \rightarrow L_{i+1} \rightarrow N_{i} \rightarrow M_{i} \rightarrow L_{i} \rightarrow N_{i-1} \rightarrow \cdots \text {. }
$$

This shows that when $k=1, M_{i}=0$ if $i \neq d-1, d-h$. Since in this case $\operatorname{dim} M=d-1$, we have $\operatorname{dim} M_{d-1}=d-1$. If $h=1$, then $M_{i} \neq 0$ if and only if $i=d-1$. When $h>1$, because $M$ contains a submodule of dimension $d-h$, we have $\operatorname{dim} M_{d-h}=d-h$. No matter what $h$ is, we have $|\Delta(M ; i)| \leq 1$ for $0 \leq i \leq n$. Thus, the claim is true by Proposition (2.3).

When $k>1$, we have

$$
N_{i} \simeq\left\{\begin{array}{cc}
0 & \text { if } i<d-1, \\
\frac{R}{\sum_{l} f_{l} R} & \text { if } i=d-1, \\
\text { some } d \text {-dimensional module, } & \text { if } i=d .
\end{array}\right.
$$

If $h=1$, then $M_{i}=0$ for $i<d-1$, and the claim follows from Proposition (2.3). Thus, we may assume that $h>1$, and in this case, from $(* *)$ we get

$$
M_{i} \simeq \begin{cases}\text { some } d \text {-dimensional module } & \text { if } i=d, \\ N_{d-1} & \text { if } i=d-1, \\ L_{d-h} & \text { if } i=d-h, \\ 0 & \text { otherwise. }\end{cases}
$$

Again, for $i \neq d-1$, the result follows from Proposition (2.3). Now we can concentrate on the case $i=d-1$, which we treat separately in Lemma (4.3).

(4.2) Definition. Let $R$ be any ring, $I, J \subset R$ be ideals, and let $a \in R$. Then

$$
I:_{R} J \stackrel{\text { def }}{=}\{r: r J \subseteq I\} \quad \text { and } \quad I:_{R} a \stackrel{\text { def }}{=} I:_{R} a R .
$$

We can simply write $I: J$ and $I: a$ when $R$ is understood.

(4.3) Lemma. Let $R=K\left[\left[x_{1}, \ldots, x_{d}\right]\right]$ be a formal power series ring of characteristic $p$. Let $\mathrm{K}_{\bullet}=\mathrm{K}_{\bullet}\left(x_{1}, \ldots, x_{d} ; R\right)$ and $\mathrm{K}^{\bullet}=\mathrm{K}^{\bullet}\left(x_{1}, \ldots, x_{d} ; R\right)$. Suppose $a_{1}, \ldots$, $a_{h}, f_{1}, \ldots, f_{k} \in R-\{0\}$. Let $A$ be the $(h \times k)$-matrix $\left(a_{l} f_{m}\right)$. Let $M=\operatorname{Coker} A$. Then the limit

$$
\lim _{e \rightarrow \infty} \frac{\ell\left(\mathrm{H}^{d-1}\left(\mathrm{~F}^{e}\left(\mathrm{~K}^{\bullet}\right) \otimes M\right)\right)}{q^{\delta^{d-1}}}
$$


exists and is equal to

$$
\sum_{j=0}^{d-1} \lim _{e \rightarrow \infty} \frac{\ell\left(\mathrm{H}_{d-1-j}\left(\mathrm{~F}^{e}\left(\mathrm{~K}_{\bullet}\right) \otimes M_{j}\right)\right)}{q^{\delta_{M}^{d-1}}} .
$$

Proof. We will follow the same notation as in Example (4.1). When $k=1$, we have $\operatorname{dim} M=d-1$, and the result is trivial. We now assume that $k>1$.

Let $I=\sum_{l} a_{l} R$ and $J=\sum_{m} f_{m} R$.

Since $L=R / I \hookrightarrow M$, if $\operatorname{dim} R / I=d-1$ then $M$ contains a submodule of dimension $d-1$, and the lemma follows from Corollary (2.4). We will assume that $\operatorname{dim} R / I<d-1$.

From (**) in Example (4.1) it follows that

$$
M_{i} \simeq \begin{cases}N_{d-1}=\frac{R}{J} & \text { if } i=d-1, \\ 0 & \text { if } \operatorname{dim} R / I<i<d-1, \\ L_{i} & \text { if } i \leq \operatorname{dim} R / I .\end{cases}
$$

This says that $\operatorname{dim} M_{d-1}=\operatorname{dim} R / J$, $\operatorname{dim} M_{\operatorname{dim} R / I}=\operatorname{dim} R / I$, and otherwise $\operatorname{dim} M_{i}<\operatorname{dim} R / I$. When $\operatorname{dim} R / J \neq \operatorname{dim} R / I$, we have $|\Delta(M ; d-1)|=1$, and the result follows from Proposition (2.3). Therefore, we may assume that $\operatorname{dim} R / J=\operatorname{dim} R / I=a$, and in this situation $\Delta(M ; d-1)=\{a, d-1\}$.

Since $\delta_{N}^{d-2}=-\infty$, the short exact sequence $(*)$ in Example 3.1 .8 induces a long exact sequence

$$
\begin{aligned}
0 \rightarrow \mathrm{H}^{d-1}\left(\mathrm{~F}^{e}\left(\mathrm{~K}^{\bullet}\right) \otimes L\right) & \rightarrow \mathrm{H}^{d-1}\left(\mathrm{~F}^{e}\left(\mathrm{~K}^{\bullet}\right) \otimes M\right) \\
& \rightarrow \mathrm{H}^{d-1}\left(\mathrm{~F}^{e}\left(\mathrm{~K}^{\bullet}\right) \otimes N\right) \stackrel{\alpha_{e}}{\longrightarrow} \mathrm{H}^{d}\left(\mathrm{~F}^{e}\left(\mathrm{~K}^{\bullet}\right) \otimes L\right) \rightarrow \cdots
\end{aligned}
$$

This implies that

$$
\begin{aligned}
& \ell\left(\mathrm{H}^{d-1}\left(\mathrm{~F}^{e}\left(\mathrm{~K}^{\bullet}\right) \otimes M\right)\right) \\
& \quad=\ell\left(\mathrm{H}^{d-1}\left(\mathrm{~F}^{e}\left(\mathrm{~K}^{\bullet}\right) \otimes L\right)\right)+\ell\left(\mathrm{H}^{d-1}\left(\mathrm{~F}^{e}\left(\mathrm{~K}^{\bullet}\right) \otimes N\right)\right)-\ell\left(\operatorname{Im} \alpha_{e}\right) .
\end{aligned}
$$

Because $\operatorname{dim} L=a$, we know that the limit

$$
\lim _{e \rightarrow \infty} \frac{\ell\left(\mathrm{H}^{d-1}\left(\mathrm{~F}^{e}\left(\mathrm{~K}^{\bullet}\right) \otimes L\right)\right)}{q^{a}}
$$

exists. Note that we also have $\Delta(N ; d-1)=\{d-1\}$ and $\delta_{N}^{d-1}=a$, so

$$
\lim _{e \rightarrow \infty} \frac{\ell\left(\mathrm{H}^{d-1}\left(\mathrm{~F}^{e}\left(\mathrm{~K}^{\bullet}\right) \otimes N\right)\right)}{q^{a}}
$$


exists. Suppose that $\ell\left(\operatorname{Im} \alpha_{e}\right)=O\left(q^{a-1}\right)$. Then

$$
\begin{aligned}
\lim _{e \rightarrow \infty} & \frac{\ell\left(\mathrm{H}^{d-1}\left(\mathrm{~F}^{e}\left(\mathrm{~K}^{\bullet}\right) \otimes M\right)\right)}{q^{a}} \\
& =\lim _{e \rightarrow \infty} \frac{\ell\left(\mathrm{H}^{d-1}\left(\mathrm{~F}^{e}\left(\mathrm{~K}^{\bullet}\right) \otimes L\right)\right)}{q^{a}}+\lim _{e \rightarrow \infty} \frac{\ell\left(\mathrm{H}^{d-1}\left(\mathrm{~F}^{e}\left(\mathrm{~K}^{\bullet}\right) \otimes N\right)\right)}{q^{a}},
\end{aligned}
$$

since $\Delta(L ; d-1)=\{a\}$ and $\Delta(N ; d-1)=\{d-1\}$,

$$
\begin{aligned}
& =\lim _{e \rightarrow \infty} \frac{\ell\left(\mathrm{H}_{d-1-a}\left(\mathrm{~F}^{e}\left(\mathrm{~K}_{\bullet}\right) \otimes L_{a}\right)\right)}{q^{a}}+\lim _{e \rightarrow \infty} \frac{\ell\left(\mathrm{H}_{0}\left(\mathrm{~F}^{e}\left(\mathrm{~K}_{\bullet}\right) \otimes N_{d-1}\right)\right)}{q^{a}} \\
& =\lim _{e \rightarrow \infty} \frac{\ell\left(\mathrm{H}_{d-1-a}\left(\mathrm{~F}^{e}\left(\mathrm{~K}_{\bullet}\right) \otimes M_{a}\right)\right)}{q^{a}}+\lim _{e \rightarrow \infty} \frac{\ell\left(\mathrm{H}_{0}\left(\mathrm{~F}^{e}\left(\mathrm{~K}_{\bullet}\right) \otimes M_{d-1}\right)\right)}{q^{a}}, \quad \text { by (\#) }
\end{aligned}
$$

and finally, because $\Delta(M ; d-1)=\{a, d-1\}$,

$$
=\sum_{j=0}^{d-1} \lim _{e \rightarrow \infty} \frac{\ell\left(\mathrm{H}_{d-1-j}\left(\mathrm{~F}^{e}\left(\mathrm{~K}_{\bullet}\right) \otimes M_{j}\right)\right)}{q^{a}} .
$$

Hence it remains to show $\ell\left(\operatorname{Im} \alpha_{e}\right)=O\left(q^{a-1}\right)$.

Since $\mathrm{H}^{d-1}\left(\mathrm{~F}^{e}\left(\mathrm{~K}^{\bullet}\right) \otimes-\right) \simeq \operatorname{Tor}_{1}^{R}\left(R / m^{[q]},-\right)$ for any $R$-module, we can compute it by resolving $M$. The following is a commutative diagram in which the rows are free resolutions of $N, M$ and $L$ respectively:

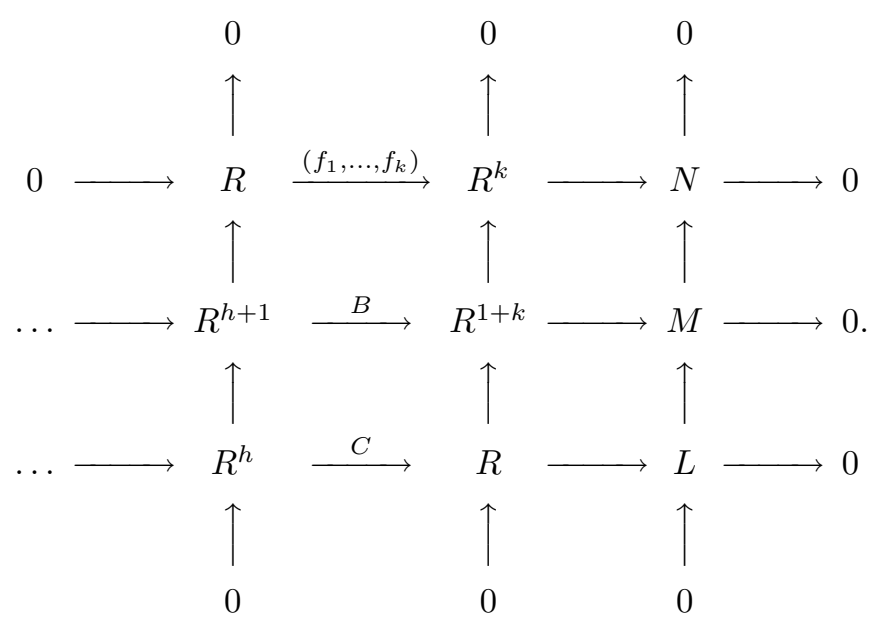

Here, $C=\left(\begin{array}{c}a_{1} \\ \vdots \\ a_{h}\end{array}\right)$. Remember that the class of 1 in $L$ is mapped to the class of $\left(f_{1}, \ldots, f_{k}\right)$ in $M$, so it can be checked that

$$
B=\left(\begin{array}{cccc}
a_{1} & & & \\
\vdots & & 0 & \\
a_{h} & & & \\
-1 & f_{1} & \cdots & f_{k}
\end{array}\right)
$$


By applying $\left(R / m^{[q]}\right) \otimes$ to the diagram, we can find out what $\operatorname{Im} \alpha_{e}$ is. The cycles $\bar{r}$ in $\operatorname{Tor}_{1}^{R}\left(R / m^{[q]}, N\right)$ are exactly those $\bar{r}$ in $R / m^{[q]}$ that satisfy $\bar{r} J \equiv 0\left(\bmod m^{[q]}\right)$. The cycle $\bar{r}$ is mapped to the class represented by $-r$ in $\operatorname{Tor}_{0}^{R}\left(R / m^{[q]}, L\right)$. Hence,

$$
\begin{aligned}
\operatorname{Im} \alpha_{e} & =\frac{\left(m^{[q]}: J\right)\left(R / m^{[q]}\right)}{I\left(R / m^{[q]}\right) \cap\left(\left(m^{[q]}: J\right)\left(R / m^{[q]}\right)\right)} \\
& \simeq \frac{\left(\left(m^{[q]}: J\right)+I\right)\left(R / m^{[q]}\right)}{I\left(R / m^{[q]}\right)} \\
& \simeq \frac{\left(m^{[q]}: J\right)+I}{m^{[q]}+I} .
\end{aligned}
$$

Now the lemma follows from Lemma (4.4) below.

(4.4) Lemma. Suppose $(R, m)$ is a regular local ring of characteristic $p$. Let $I \subset R$ be a nonzero ideal and $a \in R-\{0\}$. Then

$$
\ell\left(\frac{\left(m^{[q]}: a\right)+I}{m^{[q]}+I}\right)=O\left(q^{\operatorname{dim} R / I-1}\right)
$$

In particular, if $I, J$ are both nonzero ideals of $R$, then

$$
\ell\left(\frac{\left(m^{[q]}: J\right)+I}{m^{[q]}+I}\right)=O\left(q^{\operatorname{dim} R / I-1}\right)
$$

Proof. We can replace $a$ by a nonzero multiple of $a$ in $I$, since this will only enlarge the module we are looking at. We may assume that $a \in I$.

Let $\operatorname{dim} R=d$ and let height $I=h=d-\operatorname{dim} R / I$. Because $R$ is regular, we have $\operatorname{depth} I=$ height $I=h$. Expand $a$ to $a=a_{1}, \ldots, a_{h}$, a regular sequence in $I$. We can replace $I$ by $\left(a_{1}, \ldots, a_{h}\right) R$, for this will only enlarge the length in question. We may assume that $I=\left(a_{1}, \ldots, a_{h}\right) R$.

Let $I_{0}=\left(a_{2}, \ldots, a_{h}\right) R$ and $M=R /\left(a_{1} I+I_{0}\right)$. The map from $M$ to $R / I$ sending the class of 1 in $M$ to the class of 1 in $R / I$ is surjective, and its kernel is

$$
\frac{I}{a_{1} I+I_{0}} \simeq \frac{a_{1} R}{a_{1} R \cap\left(a_{1} I+I_{0}\right)}=\frac{a_{1} R}{a_{1} I} \simeq \frac{R}{I} .
$$

Write $N=R / I$. Then we have the short exact sequence $0 \rightarrow N \rightarrow M \rightarrow N \rightarrow 0$. For each $e$, this short exact sequence induces a long exact sequence

$$
\begin{aligned}
\cdots \rightarrow \mathrm{H}^{d-1}\left(\mathrm{~F}^{e}\left(\mathrm{~K}^{\bullet}\right) \otimes N\right) \stackrel{\alpha_{e}}{\rightarrow} \mathrm{H}^{d}\left(\mathrm{~F}^{e}\left(\mathrm{~K}^{\bullet}\right) \otimes N\right) & \\
\rightarrow & \mathrm{H}^{d}\left(\mathrm{~F}^{e}\left(\mathrm{~K}^{\bullet}\right) \otimes M\right) \rightarrow \mathrm{H}^{d}\left(\mathrm{~F}^{e}\left(\mathrm{~K}^{\bullet}\right) \otimes N\right) \rightarrow 0 .
\end{aligned}
$$

Since $M$ and $N$ both have dimension $d-h$, by Lemma (4.5) we have $\ell\left(\operatorname{Im} \alpha_{e}\right)=$ $O\left(q^{d-h-1}\right)$.

Since $R$ is regular, $\mathrm{H}^{i}\left(\mathrm{~F}^{e}\left(\mathrm{~K}^{\bullet}\right) \otimes-\right)=\operatorname{Tor}_{d-i}\left(R / m^{[q]},-\right)$. We can calculate $\mathrm{H}^{i}\left(\mathrm{~F}^{e}\left(\mathrm{~K}^{\bullet}\right) \otimes M\right)$ and $\mathrm{H}^{i}\left(\mathrm{~F}^{e}\left(\mathrm{~K}^{\bullet}\right) \otimes N\right)$ by resolving $M$ and $N$. The following is a commutative diagram whose top and bottom rows are $\mathrm{K}_{\bullet}\left(a_{1}, \ldots, a_{h} ; R\right)$ and whose 
middle row is a free resolution of $M$ :

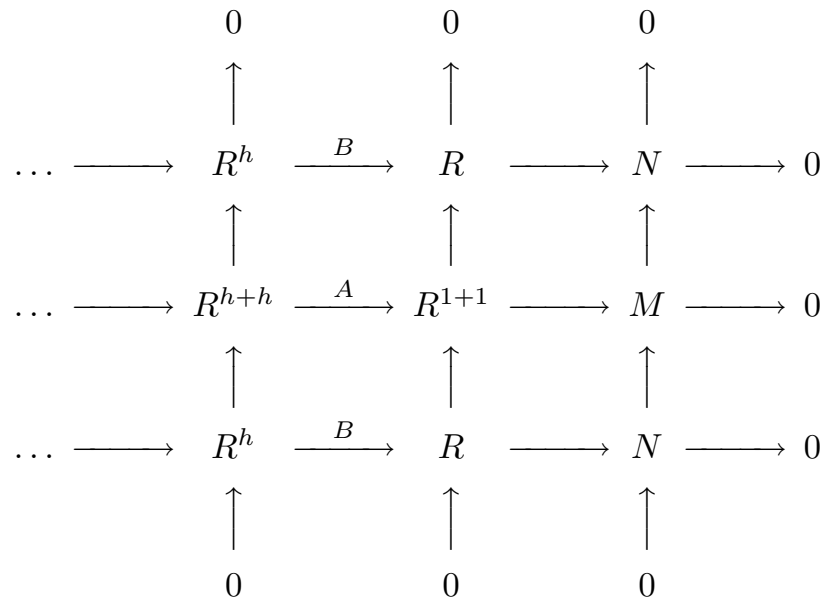

where $B=\left(\begin{array}{c}a_{1} \\ \vdots \\ a_{h}\end{array}\right)$, and it can be checked that

$$
A=\left(\begin{array}{cc}
a_{1} & \\
\vdots & \mathbf{0} \\
a_{h} & \\
-1 & a_{1} \\
\mathbf{0} & \vdots \\
& a_{h}
\end{array}\right)
$$

Apply $R / m^{[q]} \otimes$ to this diagram. With an argument similar to the one in Lemma (4.4) we can describe $\operatorname{Im} \alpha_{e}$. Let $S_{e}=\left(m^{[q]}+I_{0}\right): a$. Then $\operatorname{Im} \alpha_{e}$ is exactly

$$
\frac{S_{e}+I}{m^{[q]}+I} \text {. }
$$

Note that $m^{[q]}: a \subset S_{e}$. Hence

$$
\ell\left(\frac{m^{[q]}: a+I}{m^{[q]}+I}\right) \leq \ell\left(\operatorname{Im} \alpha_{e}\right)=O\left(q^{d-h-1}\right) .
$$

(4.5) Lemma. Let $R$ be any local ring of characteristic $p$ and $G^{\bullet}$ be a finite free complex of finite length homology. Let $M, M^{\prime}$ and $M^{\prime \prime}$ be finitely generated modules of dimension no more than d such that $0 \rightarrow M^{\prime} \rightarrow M \rightarrow M^{\prime \prime} \rightarrow 0$ is a short exact sequence. Then for each $i$,

$$
\begin{aligned}
\lim _{e \rightarrow \infty} & \frac{\ell\left(\mathrm{H}^{i}\left(\mathrm{~F}^{e}\left(G^{\bullet}\right) \otimes M\right)\right)}{q^{d}} \\
& =\lim _{e \rightarrow \infty} \frac{\ell\left(\mathrm{H}^{i}\left(\mathrm{~F}^{e}\left(G^{\bullet}\right) \otimes M^{\prime}\right)\right)}{q^{d}}+\lim _{e \rightarrow \infty} \frac{\ell\left(\mathrm{H}^{i}\left(\mathrm{~F}^{e}\left(G^{\bullet}\right) \otimes M^{\prime \prime}\right)\right)}{q^{d}} .
\end{aligned}
$$

Proof. See [Sei, Proposition 1(b)]. 


\section{REFERENCES}

[BE] D. Buchsbaum and D. Eisenbud, What makes a complex exact? J. of Algebra 25 (1973), 259-268. MR 47:3369

[Cc] A. Conca, Hilbert-Kunz function of monomial ideals and binomial hypersurfaces, preprint.

[Ct] M. Contessa, On the Hilbert-Kunz function and Koszul homology, J. of Algebra 175 (1995), 757-766; 180 (1966), 321-322. MR 96e:13018; CMP 1996:8

[D1] S. P. Dutta, Frobenius and multiplicities, J. of Algebra 85 (1983), 424-448. MR 85f:13022

[D2] _ Exact and Frobenius, J. of Algebra 127 (1989), 163-177. MR 91g:13018

[F] W. Fulton, Intersection Theory, Springer-Verlag, 1984. MR 85k:14004

[GH] A. Grothendieck (notes by R. Hartshorne), Local Cohomology, Lecture Notes in Math. No. 41, Springer-Verlag, 1967. MR 37:219

[He] J. Herzog, Ringe der Charakteristik $p$ und Frobeniusfunktoren, Math. Z. 140 (1974), 6778. MR 50:4569

[HH1] M. Hochster and C. Huneke, Tight closure, invariant theory, and the Briançon-Skoda theorem, J. A.M.S. 3 (1990), 31-116. MR 91g:13010

[HH2] — Tight closure and strong F-regularity, Mém. Soc. Math. France 38 (concré au colloque en l'honneur de P. Samuel) (1989), 119-133. MR 91i:13025

[HH3] _ Phantom homology, Mem. Amer. Math. Soc. 103 (1993), no. 490. MR 93j:13020

[HH4] $\quad$ F-regularity, test elements and smooth base change, Trans. Amer. Math. Soc. 346 (1994), 1-62. MR 95d:13007

[HM] C. Han and P. Monsky, Some surprising Hilbert-Kunz functions, Math. Z. 214 (1993), 119-135. MR 94f: 13008

[K1] E. Kunz, Characterizations of regular local rings for characteristic $p$, Amer. J. Math. 91 (1969), 772-784. MR 40:5609

[K2] , On Noetherian rings of characteristic p, Amer. J. Math. 98 (1976), 999-1013. MR 55:5612

[Mac] S. MacLane, Homology, Classics in Mathematics, Springer-Verlag, 1995. MR 96d:18001

[Mat1] H. Matsumura, Commutative Algebra, Benjamin/Cummings, Reading, Ma., second edition, 1980. MR 82i: 13003

[Mat2] — Commutative Ring Theory, Cambridge Univ. Press, 1986. MR 88h:13001

[Mo1] P. Monsky, The Hilbert-Kunz function, Math. Ann. 263 (1983), 43-49. MR 84k:13012

[Mo2] - Fine estimates for the growth of $e_{n}$ in $\mathbb{Z}_{p}^{d}$-extensions, Algebraic Number Theory (I. Iwasawa 70th Birthday Vol.), Adv. Stud. Pure Math., vol. 17, Academic Press, Boston, MA, and Kinokuniya, Tokyo, 1989, pp. 309-330. MR 90e:11120

[N] D. G. Northcott, Finite Free Resolutions, Cambridge Univ. Press, 1976. MR 57:377

[PS] C. Peskine and L. Szpiro, Dimension projective finie et cohomologie locale, Inst. Hautes Études Sci. Publ. Math. 42 (1973), 47-119. MR 51:10330

[R1] P. Roberts, Le théorème d'intersection, C. R. Acad. Sci. Paris Sér. I Math. 304 (1987), 177-180. MR 89b:14008

[R2] - Intersection theorems, Commutative Algebra, (M. Hochster et al., eds.), Math. Sci. Res. Inst. Publ., vol. 15, Springer-Verlag, 1989, pp. 417-436. MR 90j:13024

[Sei] G. Seibert, Complexes with homology of finite length and Frobenius functors, J. of Algebra 125 (1989), 278-287. MR 90j:13012

[Ser] J.-P. Serre, Algèbre locale. Multiplicités, Lecture Notes in Math., no. 11, Springer-Verlag, 1965. MR 34:1352

[St] J. Strooker, Homological Questions in Local Algebra, London Math. Soc. Lecture Note Ser., No. 145, Cambridge Univ. Press, 1990. MR 91m:13013

[Sz] L. Szpiro, Sur la théorie des complexes parfaits, Commutative Algebra (Durham, 1981), London Math. Soc. Lecture Note Ser., No. 72, Cambridge Univ. Press, 1982, pp. 83-90. MR 84m: 13015

Department of Mathematics, National Chung Cheng University, Minghsiung, Chiayi 621, TAIWAN, R. O. C.

E-mail address: stchang@math.ccu.edu.tw 